\title{
Deriving stellar parameters from GALANTE photometry: bias and precision
}

\author{
A. Lorenzo-Gutiérrez, ${ }^{1 \star}$ E. J. Alfaro, ${ }^{1,2}$ J. Maíz Apellániz ${ }^{\oplus},{ }^{3}$ R. H. Barbá ${ }^{\oplus},{ }^{4}$ \\ A. Marín-Franch, ${ }^{2,5}$ A. Ederoclite, ${ }^{2,5,6}$ D. Cristóbal-Hornillos, ${ }^{2,5}$ J. Varela, ${ }^{2,5}$ \\ H. Vázquez Ramió, ${ }^{2,5}$ A. J. Cenarro, ${ }^{2,5}$ D. J. Lennon, ${ }^{7}$ P. García-Lario, ${ }^{7}$ S. Daflon ${ }^{8}$ \\ and M. Borges Fernandes ${ }^{8}$ \\ ${ }^{1}$ Instituto de Astrofísica de Andalucía, Glorieta de la Astronomía s/n, E-18008 Granada, Spain \\ ${ }^{2}$ Unidad Asociada CEFCA-IAA, CSIC, E-44001 Teruel, Spain \\ ${ }^{3}$ Centro de Astrobiología, CSIC-INTA, Campus ESAC, Camino bajo del castillo s/n, E-28692 Villanueva de la Cañada, Spain \\ ${ }^{4}$ Universidad de La Serena, La Serena 1700000, Chile \\ ${ }^{5}$ Centro de Estudios de Física del Cosmos de Aragón, E-44001 Teruel, Spain \\ ${ }^{6}$ Instituto de Astronomia, Geofísica e Ciências Atmosféricas, Universidade de São Paulo, 05508-090 Brazil \\ ${ }^{7}$ European Space Agency, ESAC, Camino bajo del castillo s/n, E-28692 Villanueva de la Cañada, Madrid, Spain \\ ${ }^{8}$ Observatòrio Nacional, Rua Gal. José Cristino 77, 20921-400, Rio de Janeiro, RJ, Brazil
}

Accepted 2020 March 24. Received 2020 March 23; in original form 2019 September 2

\begin{abstract}
In this paper, we analyse how to extract the physical properties from the GALANTE photometry of a stellar sample. We propose a direct comparison between the observational colours (photometric bands normalized to the $515 \mathrm{~nm}$ central wavelength) and the synthetic colours derived from different stellar libraries. We use the reduced $\chi^{2}$ as the figure of merit for selecting the best fitting between both colour sets. The synthetic colours of the Next Generation Spectral Library (NGSL) provide a valuable sample for testing the uncertainty and precision of the stellar parameters derived from observational data. Reddening, as an extrinsic stellar physical parameter becomes a crucial variable for accounting for the errors and bias in the derived estimates: the higher the reddenings, the larger the errors and uncertainties in the derived parameters. NGSL colours also enable us to compare different theoretical stellar libraries for the same set of physical parameters, where we see how different catalogues of models can provide very different solutions in a, sometimes, non-linear way. This peculiar behaviour makes us to be cautious with the derived physical parameters obtained from GALANTE photometry without previous detailed knowledge of the theoretical libraries used to this end. In addition, we carry out the experiment of deriving physical stellar parameters from some theoretical libraries, using some other libraries as observational data. In particular, we use the Kurucz and Coelho libraries, as input observational data, to derive stellar parameters from Coelho + TLUSTY and Kurucz + TLUSTY stellar libraries, respectively, for different photometric errors and colour excesses.
\end{abstract}

Key words: techniques: photometric-stars: formation-stars: fundamental parametersgalaxies: star formation.

\section{INTRODUCTION}

Getting intrinsic stellar physical parameters such as effective temperature, gravity, and metallicity, as well as extrinsic reddening, is a primary aim for any photometric system. The GALANTE

^E-mail: alorenzogutierrez@gmail.com system is not an exception. In this paper, we analyse how to get the physical stellar properties from the GALANTE photometry. To do this, we make use of different theoretical and observational stellar libraries. GALANTE is a new photometric system specially designed to carry out a mapping of the bright star population of the northern Galactic plane $(V \leq 17)$. It is defined by seven filters in the optical range, three intermediate-broad and four narrow (see fig. 1b in Lorenzo-Gutiérrez et al. 2019). This configuration makes 
it possible an accurate determination of the Balmer jump, the $\mathrm{H}_{\alpha}$ line and the Calcium II triplet. The performances of the filters can be seen in Lorenzo-Gutiérrez et al. (2019). Thus, we expect to characterize the bright star population of the Galactic disc. Another objective is to build a spectral energy distribution (SED) catalogue to substitute Tycho to a certain extent for a complete coverage of the optical range of the disc's bright stars. The system and the mapping (bearing the same name) are being conducted using the Cassegraintype T80 telescope at the Javalambre Observatory (Teruel, Spain). The telescope, with a focal length of $3712 \mathrm{~mm}$, covers a field of view of $1.7 \mathrm{sq}$ deg free from vignetting.

Selection of this filter set allows us to get reliable, valuable information on the atmospheric parameters of the stars (Maíz Apellániz 2017). The GALANTE project presents some peculiarities that distinguish it from other surveys based on different coverage and sampling in the optical range. Some of these differences are mainly based on the observed region of the sky and the limit of magnitude, obtaining complete photometry to $V=17$, but reaching up to 18 19 in many regions of the Galactic disc. On the other hand, the definition of the seven GALANTE filters has been chosen to extract the maximum information about the physical properties of the giant stars in the solar neighbourhood and of the OB stars within the observed magnitude limit. GALANTE has an optimized filter set to measure the Balmer jump to determine $T_{\text {eff }}$ for hot stars. It uses the F348M and F420N filters, and it includes two narrow filters (F660N and $\mathrm{F} 665 \mathrm{~N}$ ) for measuring $\mathrm{H}_{\alpha}$ flux.

In this paper, we present an algorithm based on the GALANTE normalized colour fitting to different star libraries covering an extensive range of physical parameters. The likelihood criterium is the well-known $\chi_{\text {red }}^{2}$ (Barlow 1989), which has been used for the same purpose in a wide variety of studies (Allende Prieto \& del Burgo 2016; Bohlin et al. 2017; Rodrigo et al. 2019).

There exists a number of points to determine the physical parameters of a set of stars with a certain photometry. One of most important is the appropriate choice of the stellar libraries with which they are to be compared. Obviously, the observed stars must be within the set of values that define the parametric space of the models. However, in most cases we do not know beforehand if this condition is satisfied. Another fundamental point is the choice of the best extinction law that describes the properties of the interstellar medium in the studied region. This is particularly when we work with stars that may be highly reddened. It is well known that there are regions of the Galactic disc with peculiar dust properties, which would imply a change in the $R_{5495}$ parameter that would define the best model of the interstellar extinction curve (Maíz Apellániz \& Sota 2008; Maíz Apellániz 2013b; Maíz Apellániz et al. 2014; Maíz Apellániz \& Barbá 2018).

In this work, we analyse the capabilities of the algorithm developed to determine the physical stellar properties from the GALANTE photometry. To do this, we use the Next Generation Spectral Library (NGSL) observational spectral library (Version 2; Gregg et al. 2006) as a sample test to estimate the precision and uncertainties of the derived parameters, comparing with four different sets of stellar models that cover a wide range of spectral types, luminosity classes, and chemical composition. This analysis provides us information about the best selection of models for the obtaining of physical stellar parameters free from bias and with better internal precision. We want to answer, for example, whether it is better to compare the observations with the whole set of available stellar models or if, on the contrary, a previous selection is needed to ensure a better result. Thus, we focus this study on the development and description of an algorithm for obtaining the physical stellar properties and the analysis of how different libraries of stellar models can give different results, introducing, in some cases, a considerable bias.

We have organized the paper into five sections, the first being this introduction. In Section 2, we describe the determination of the GALANTE normalized colours from the NGSL observational spectra and the stellar models, and we analyse the different properties of the GALANTE SEDs derived from the different models used. The fitting algorithm, called MASTER (Monte Carlo Astrophysics Studio for galanTE colouRs), is described in Section 2.3. Section 3 is assigned to comparing two libraries of models that cover almost completely the same range of stellar physical parameters using MASTER to determine the physical properties derived for a library using the other as a comparison model. This experiment, which considers different internal precisions, allows us to quantify the degree of uncertainty introduced by the methodology itself and the use of different stellar models, as well as different values of reddening. In Section 4, we use the observational spectral library NGSL to analyse the goodness of the results, using four sets of theoretical models in different combinations of them, as the reference for obtaining the main physical properties. Finally, in Section 5, we summarize the main results and state the conclusions of this study.

\section{DATA AND METHODS}

\subsection{Libraries}

\subsubsection{Theoretical stellar libraries}

The GALANTE project is mainly designed for the study of the Galactic disc (Maíz Apellániz 2017; Lorenzo-Gutiérrez et al. 2019; Maíz Apellániz et al. 2019). Thus, the set of theoretical models that will be used to determine the physical parameters of the sample objects should be focused mainly on the most characteristic stars of this Galactic subsystem. To this end, we select the Coelho low-resolution (hereinafter C, Coelho 2014), Kurucz (hereinafter K, Castelli, Gratton \& Kurucz 1997), and TLUSTY OSTAR2002 (hereinafter T, Lanz \& Hubeny 2003) theoretical libraries. C's library covers $T_{\text {eff }}$ from 3000 to $25000 \mathrm{~K}$ (in steps of $200 \mathrm{~K}$ below $T_{\text {eff }}=4000$ and $1000 \mathrm{~K}$ above $T_{\text {eff }}=12000$ and $250 \mathrm{~K}$ otherwise), with $\log g$ between 0.5 and 5.5 (in steps of $0.5 \mathrm{dex}$ ) and $[\mathrm{Fe} / \mathrm{H}]$ from -1.3 to 0.2 , modelling these parameters for two $\alpha$-enhanced values ( 0.0 and 0.4 ), and for wavelengths between 1300 and 10000 $\AA$. K's catalogue provides a coverage of $3500 \mathrm{~K} \leq T_{\text {eff }} \leq 50000 \mathrm{~K}$ (in steps of $200 \mathrm{~K}$ below $T_{\text {eff }}=13000$ and $1000 \mathrm{~K}$ otherwise), $0.0 \leq \log g \leq 5.0$ dex (in steps of $0.5 \mathrm{dex}$ ) and $-2.5 \leq[\mathrm{Fe} / \mathrm{H}] \leq$ 0.5 (in steps of 0.2 and $0.5 \mathrm{dex}$ ). The range of wavelengths in $\mathrm{K}$ is from $1000 \AA$ to $10 \mu \mathrm{m}$ for low-resolution spectra. Both sets of models, $\mathrm{C}$ and $\mathrm{K}$, are based on the calculation of atmospheres by ATLAS9 (Kurucz 1970, 2005), or its descendants (ATLAS12, SYNTHE, and DSYNTHE), which use different opacity tables for each case. Perhaps, the greatest difference between the $\mathrm{C}$ and $\mathrm{K}$ models used in this study is based on the range of temperatures below $4000 \mathrm{~K}$ : $\mathrm{C}$ uses the MARCS code, which includes new molecular species, and $\mathrm{K}$ utilizes ATLAS9. T is another library that especially covers $\mathrm{O}$ stars, ${ }^{1}$ and that therefore enables a better redefinition of the space of parameters for the higher temperatures. The temperature range

${ }^{1}$ http://svo2.cab.inta-csic.es/theory/newov/index.php?model=tlusty_merge dbin 

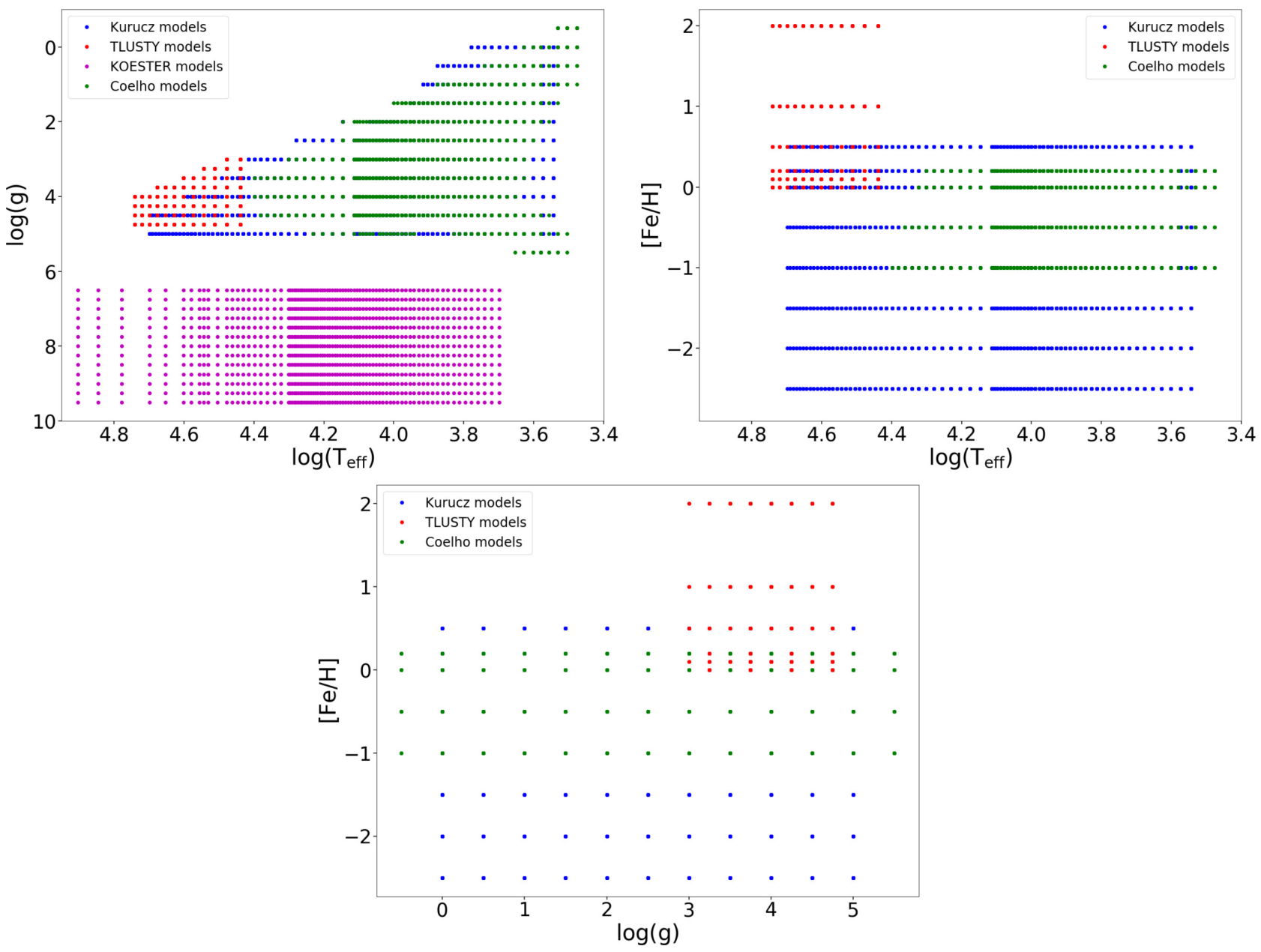

Figure 1. Range of physical stellar parameters for the theoretical libraries. Each library is coded by colours.

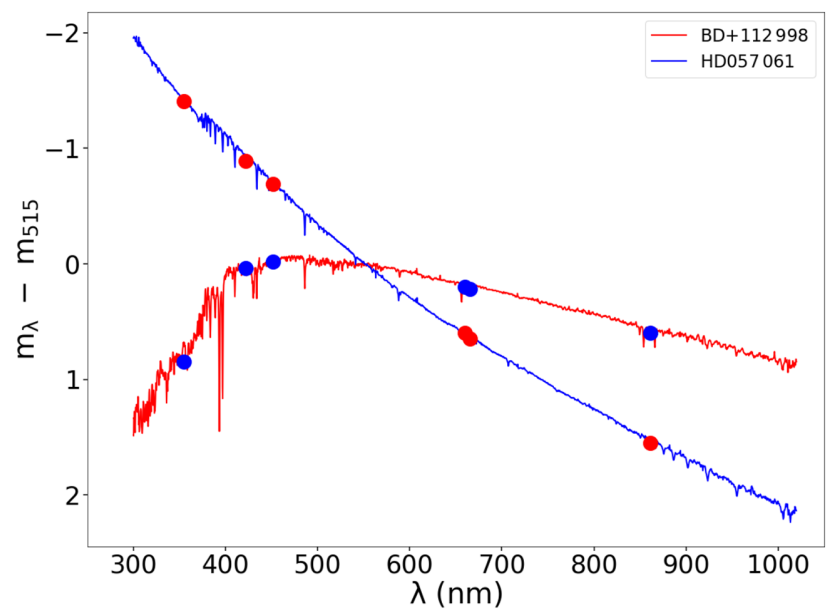

Figure 2. Two NGSL spectra in normalized magnitudes. We represent in red line BD $+112998\left(T_{\text {eff }}=5480 \mathrm{~K}\right)$ and in blue line HD057061 $\left(T_{\text {eff }}=32514 \mathrm{~K}\right)$. They are representative of the tips in the NGSL effective temperature distribution.

goes from $27500 \mathrm{~K} \leq T_{\text {eff }} \leq 55000 \mathrm{~K}$ (in steps of $2500 \mathrm{~K}$ ), $3.0 \leq$ $\log g \leq 4.75$ (in steps of $0.25 \mathrm{dex}$ ), and $[\mathrm{Fe} / \mathrm{H}]$ from 0.0 to $2.0 \mathrm{dex}$ where the accurate calibrated spectra is limited to the $3000-7500 \AA$ wavelength range. In order to extend our grid of models to another type of stars, we select the KOESTER (hereinafter Ko) white dwarf
(WD) theoretical catalogue (Koester 2010). This theoretical library contains WD spectra whose atmospherical parameters cover $T_{\text {eff }}$ from 5000 to $80000 \mathrm{~K}$ in $250 \mathrm{~K}$ steps until $20000 \mathrm{~K}$, in $1000 \mathrm{~K}$ steps from 20000 to $30000 \mathrm{~K}$, in $2000 \mathrm{~K}$ steps until $40000 \mathrm{~K}$, in 5000 and $10000 \mathrm{~K}$ steps until $80000 \mathrm{~K} . \log g$ ranges from 6.5 to 9.5 in 0.25 dex steps from 0.9 to $3000 \mathrm{~nm}$.

Fig. 1 is a graphical representation of the range of physical stellar parameters for each theoretical library.

\subsubsection{Observational stellar library: NGSL}

We have chosen the NGSL library (Gregg et al. 2006), which comprises spectra for 378 stars, covering the spectral range from 0.2 to $1.0 \mu \mathrm{m}$ well calibrated in flux. This catalogue covers a range of $3100 \leq T_{\text {eff }} \leq 32900 \mathrm{~K}, 0.4 \leq \log g \leq 5.4 \mathrm{dex}$, and $-2.0 \leq[\mathrm{Fe} / \mathrm{H}]$ $\leq 0.5$ and $E(B-V)$ from 0 to 0.75 . One can see how the physical stellar parameters of this observational library are representative of the three major subsystems of the Galaxy. Therefore, we choose this observational catalogue as input to compare with model libraries. NGSL data have been used for similar purposes in some previous studies (Aparicio Villegas et al. 2010; Mann \& von Braun 2015; Heap \& Lindler 2016; Weiler 2018). In Fig. 2, we show two NGSL spectra in normalized magnitudes as an example of SEDs that we use in this comparison with models. We represent in red line BD $+112998\left(T_{\text {eff }}=5480 \mathrm{~K}\right)$ and in blue line HD057061 $\left(T_{\text {eff }}=32514 \mathrm{~K}\right)$. Colour dots represent the GALANTE normalized 

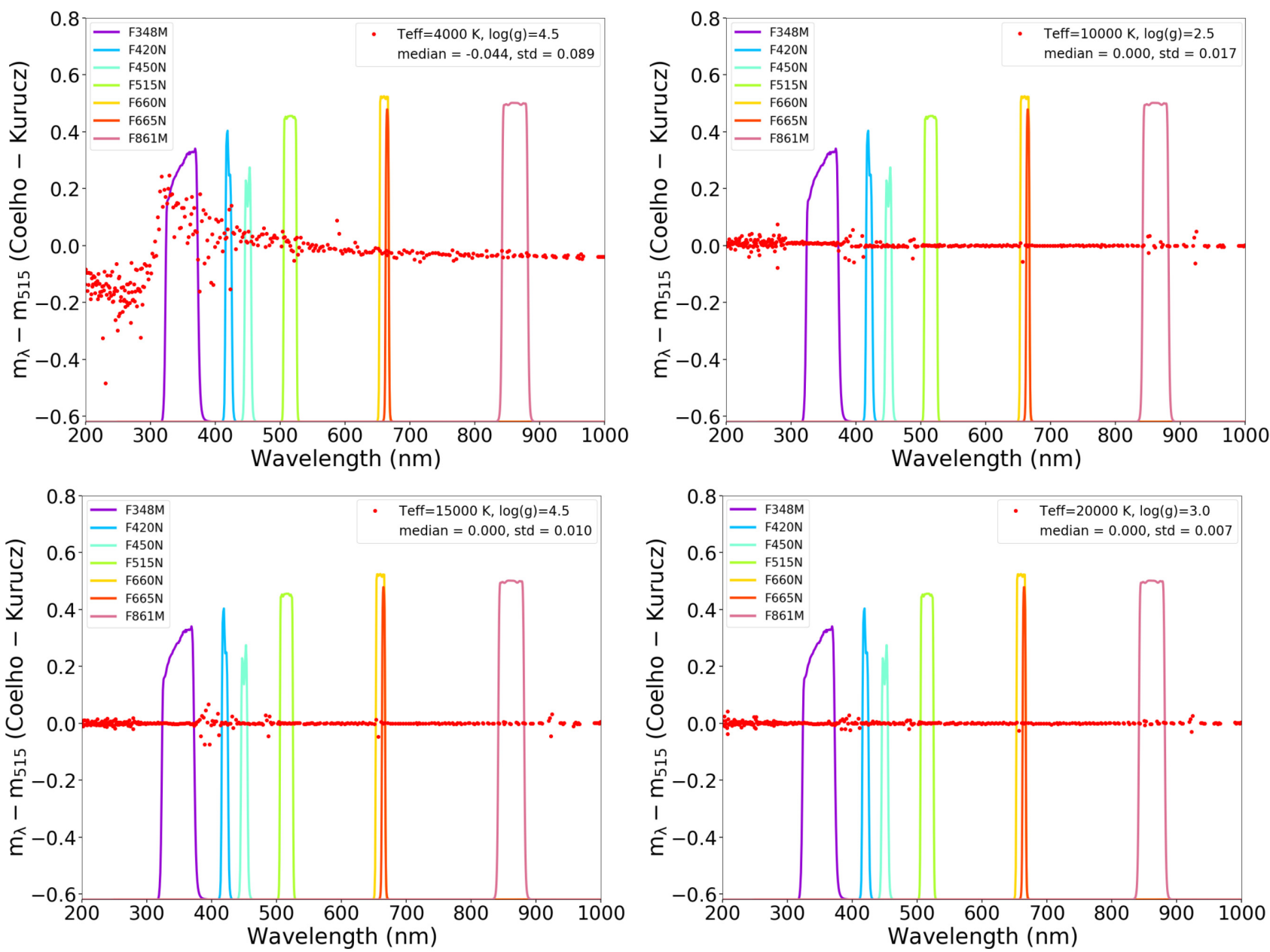

Figure 3. Red dots represent differences between $\mathrm{C}$ and $\mathrm{K}$ libraries in normalized magnitudes. Colour lines are transmission curves for GALANTE filters, which have been properly scaled to fit the plot.

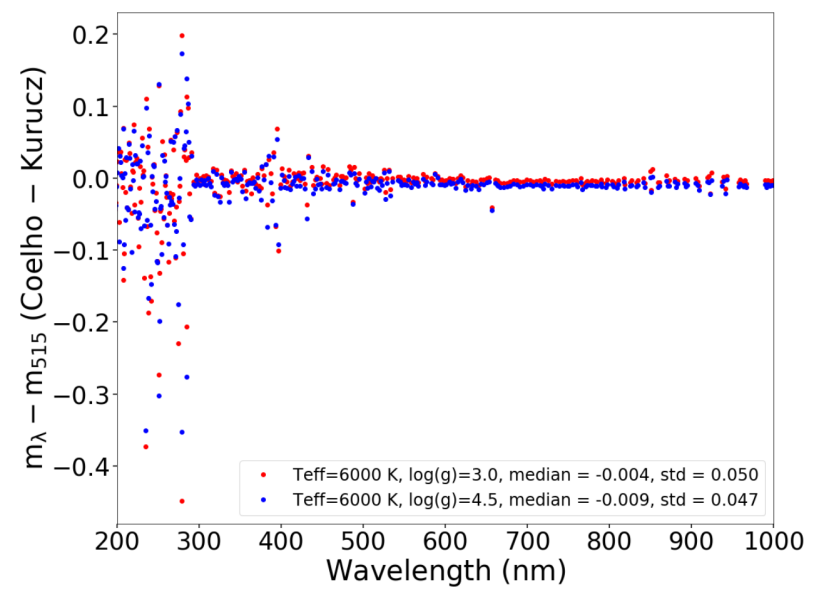

Figure 4. In blue dots, the normalized magnitude difference for an MS star with $6000 \mathrm{~K}$; and red dots represent differences for a giant star model with the same temperature.

magnitude for each NGSL spectrum. They are representative of the tips in the NGSL effective temperature distribution.

In our study, we excluded those stars catalogued as suspect by Heap \& Lindler (2016) from the NGSL library. These spectra show a poor fit with the models used by these authors to estimate their physical parameters. Those stars classified as binary by Koleva \& Vazdekis (2012) have also been removed from our analysis. Thus, our final sample consists of 251 objects that, classified by range of spectral types, are as follows: OBA ( $\left.T_{\text {eff }}>8000 \mathrm{~K}, 67 \mathrm{stars}\right)$, FGK $\left(4000<T_{\text {eff }} \leq 8000 \mathrm{~K}, 164\right.$ stars $)$, and $\mathrm{M}\left(T_{\text {eff }} \leq 4000 \mathrm{~K}\right.$, 20 stars). In this paper, we will use the physical variables listed by Koleva \& Vazdekis (2012) in their table 1, having removed the aforementioned stars.

\subsection{Magnitudes and colour calculation}

We determine the synthetic AB GALANTE magnitudes of both the theoretical and the observational libraries following the procedure described in Lorenzo-Gutiérrez et al. (2019). With these magnitudes, we build the SEDs, normalizing them to the central wavelength band $515.5 \mathrm{~nm}$. This definition of normalized SED allows us to work with different stellar models whose energy emission is given in different ways, such as brightness between the $\mathrm{C}$ and $\mathrm{K}$ models. The use of colours defined as $m_{\lambda}-m_{515}$ removes this problem. The dependence on distance when comparing the observational SEDs with theoretical models is also removed. That is, we adopted the same approach as that chosen by Allende Prieto (2016) to solve a similar problem. 

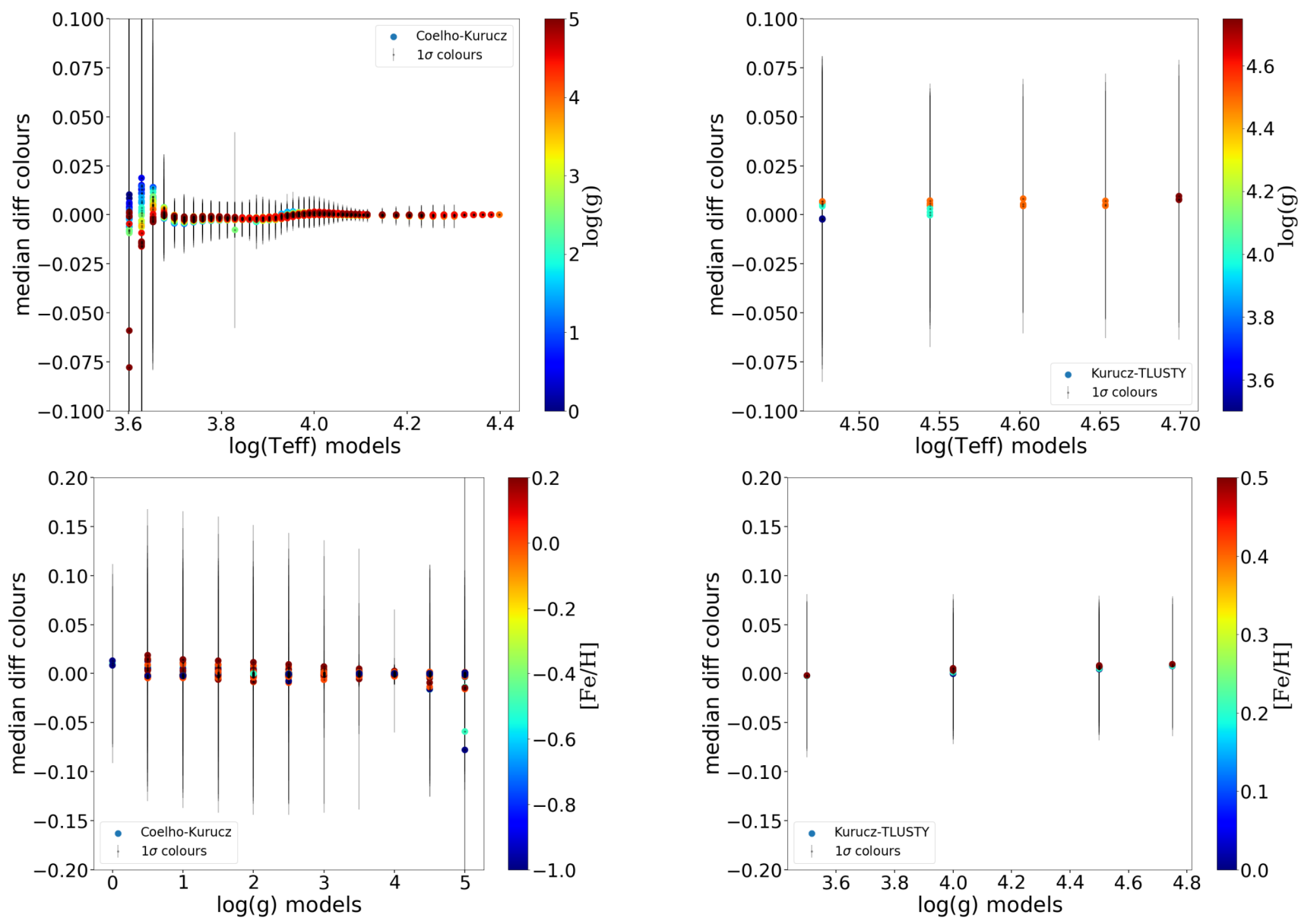

Figure 5. Differences between GALANTE normalized magnitudes for each pair of models ( $\mathrm{C}$ and $\mathrm{K})$. The top panel shows how for temperatures lower than $4500 \mathrm{~K}$ the differences are larger. In the bottom panel, we can see how metallicity is not an important factor affecting the main difference, excepting for $\log g=5.0$, where low $[\mathrm{Fe} / \mathrm{H}]$ values seem to show a negative bias with a larger dispersion.

In this paper, we will refer interchangeably to these colours as GALANTE normalized colour and GALANTE normalized magnitude.

In order to learn the properties of these theoretical libraries in depth, we carry out an analysis of the flux ratios of the different models for stellar objects with the same physical parameters. In particular, we analyse in more detail the $\mathrm{C}$ and $\mathrm{K}$ models, which cover the largest range of physical parameters. First, we choose the intersection of models with the same three physical variables, selecting them a set of 1155 spectra.

In this first exercise, we take those models with solar metallicity $[\mathrm{Fe} / \mathrm{H}]=0.0$. Fig. 3 represents the fluxes ratio in normalized magnitudes for different spectral types and luminosity classes. Superimposed on this plot, we draw the transmission curve of the different GALANTE filters.

Regarding Fig. 3, the most remarkable features can be summarized as follows: (a) the main differences are obtained for the cold stars, decreasing the bias and the error (measured by the standard deviation) as we increase the temperature; and (b) these differences are mainly found for the wavelength range corresponding to the near-ultraviolet side and other closer bands located on the other side of the Balmer jump. This plot already suggests that the determination of the observational physical parameters obtained

Figure 6. Same as Fig. 5 but for models K and T. In the top panel, we can see how the dispersion is similar for all temperatures but much larger than that reported for $\mathrm{C}$ and $\mathrm{K}$, for temperatures larger than $4500 \mathrm{~K}$. In the bottom panel, we observe that the differences do not show a clear bias.

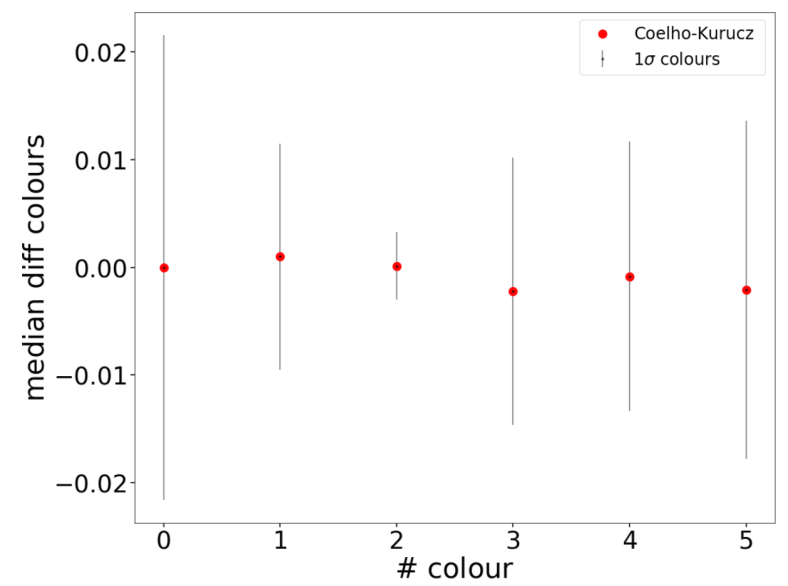

Figure 7. Differences of normalized magnitudes for each GALANTE colour between $\mathrm{C}$ and $\mathrm{K}$ common models.

from these different model catalogues could give different results, especially for low-temperature stars. In all panels of Fig. 3 we have included the median and standard deviation of these differences.

As we show in Fig. 4, the major differences in normalized magnitude are mainly due to the spectral type and not to the luminosity class, since for both a giant and a $6000 \mathrm{~K}$ main-sequence 
Table 1. Colour code.

\begin{tabular}{ll}
\hline Number of colour & GALANTE colour \\
\hline 0 & F348M - F515N \\
1 & F420N - F515N \\
2 & F450N - F515N \\
3 & F660N - F515N \\
4 & F665N - F515N \\
5 & F861M - F515N \\
\hline
\end{tabular}

(MS) star, the differences between the two models are almost identical. The comparison of models for the same luminosity class shows that this stellar parameter is not critical against the effective temperature.

For each of the 1155 models selected above, this analysis is now shifted to the SEDs defined by the GALANTE normalized colours. Here, we refer to the residual differences that are obtained as the median of the residuals for each colour.

Fig. 5 presents the differences of GALANTE normalized colours for each pair of models ( $\mathrm{C}$ and $\mathrm{K}$ ). In the top panel we observe that, for objects with $\log \left(T_{\text {eff }}\right)<3.65$ the standard deviation of the values exceeds $0.05 \mathrm{mag}$, a situation that only occurs again for models with a $T_{\text {eff }}$ close to $6000 \mathrm{~K}$. The bottom panel shows how these differences are almost invariant versus luminosity class for a wide range of metallicities. Only stars with $\log g=5.0$ show a higher dispersion since they cover the highest range in $T_{\text {eff }}$.

If we perform this same analysis with $\mathrm{K}$ and $\mathrm{T}$, we find that the differences in colours are larger for the range of temperatures in common. However, the dispersion in the differences of colours decreases for the range of $\log g$ analysed, as we can see in Fig. 6.

Regarding the differences in the GALANTE colours versus $\log g$, we see a similar effect to the previous one, but with standard deviations smaller than in the $\mathrm{C}-\mathrm{K}$ comparison. Metallicities here are not segregated in this group of models, mainly because metallicities are limited to a lesser range of values. This effect means that models are indistinguishable with this methodology as far as these parameters are concerned.

Fig. 7 shows the differences of normalized magnitudes for each GALANTE colour between $\mathrm{C}$ and $\mathrm{K}$ common models. For simplicity, Table 1 shows the correspondence between GALANTE colour and a numerical code.

It is a remarkable result that, despite the fact that all colour differences are obtained close to 0.0 , there is a systematic deviation towards negative values. This indicates that the $\mathrm{C}$ library shows values of colours somewhat higher than those obtained with the $\mathrm{K}$ catalogue. Another remarkable fact is the error of 2 per cent that occurs in the first colour (F348M - F515N). This corresponds to the bluest GALANTE band F348M, which is responsible of the measurement of the $T_{\text {eff }}$. On the contrary, the two following colours $(\mathrm{F} 420 \mathrm{~N}-\mathrm{F} 515 \mathrm{~N}$ and $\mathrm{F} 450 \mathrm{~N}-\mathrm{F} 515 \mathrm{~N})$, also involved in this estimation, are closer in the model comparison.

Attending to the $\mathrm{K}-\mathrm{T}$ model comparison, we see a systematic deviation in the bluest and the reddest optical bands of GALANTE. As we see in Fig. 8, for the bluest colour differences of 3 per cent are prevailing, dominating T. On the contrary, differences in the colour 5 (F861M - F515N) are dominated by the K library. However, we do not see deviations that differ from the observed Fig. 7, between the $\mathrm{C}$ and $\mathrm{K}$ models. The differences seen in Fig. 8 are within $0.01 \mathrm{mag}$ for all colours except for 0 and 5 . In the case of F348M - F515N, the analysis of Maíz Apellániz et al. (2014) showed that the T values for the colours associated with the Balmer jump agree with the spectroscopic values for $T_{\text {eff }}$. Thus, differences around -0.03 mag

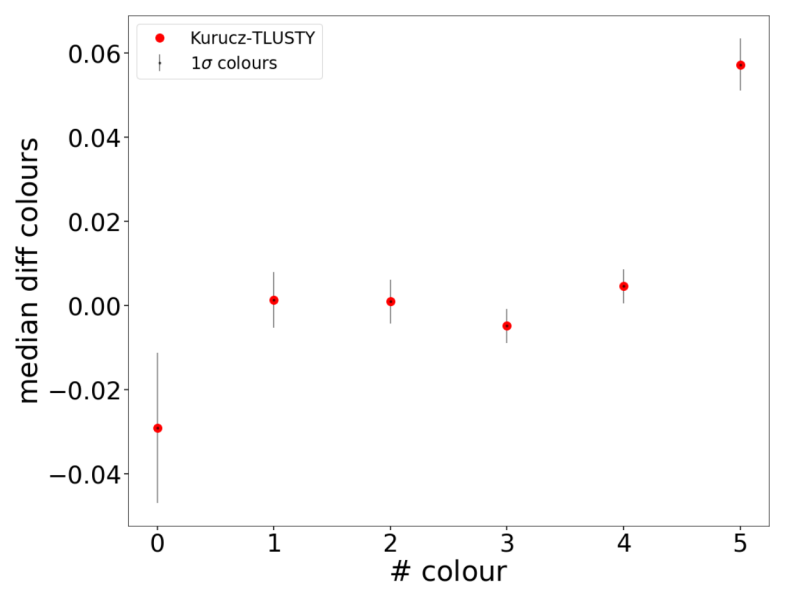

Figure 8. Differences of normalized magnitudes for each GALANTE colour between $\mathrm{K}$ and $\mathrm{T}$ common models. We can see how the colours 0 and 5 show a clear bias mainly affecting the reddest and bluest colours.

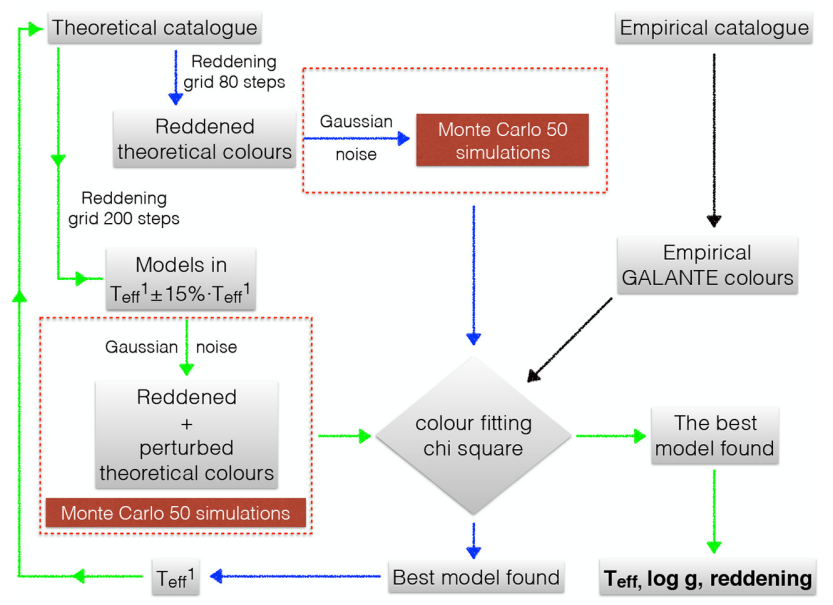

Figure 9. Flux diagram for MASTER algorithm.

are likely caused by the $\mathrm{K}$ values being incorrect. On the other hand, for F515N - F861M, the T models do not include a detailed atmospheric treatment at the wavelength range to the right of the Paschen jump, so that is the likely culprit for the difference.

We do not compare the models from $\mathrm{C}$ and $\mathrm{T}$ because there is no coincidence in effective temperatures between them.

\subsection{Fitting SEDs with MASTER}

The main idea underlying this work is to extract as much information as possible from the GALANTE photometry using theoretical and observational libraries. For this purpose, we use the GALANTE normalized colours and we obtain synthetic photometry using theoretical and empirical libraries, applying $\chi_{\text {red }}^{2}$ (hereinafter $\chi^{2}$ ) methodology to compare.

The four physical variables we want to determine using the observational GALANTE photometry are: effective temperature, gravity, metallicity, and colour excess. We present the MASTER procedure, using the previous theoretical catalogues as models libraries and the NGSL observational catalogue as input of observed stars to test them. A flux diagram of this methodology is shown in Fig. 9, where each of the steps followed in the determination of these physical variables is detailed. 
Table 2. Number of cells of each model mesh used in this work, for both the first and the second runs.

\begin{tabular}{lrcrrr}
\hline & $\mathrm{K}+\mathrm{T}$ & $\mathrm{K}+\mathrm{T}+\mathrm{Ko}$ & $\mathrm{C}+\mathrm{T}$ & $\mathrm{C}+\mathrm{T}+\mathrm{Ko}$ \\
\hline First run, cells & $83 \times 15 \times 11 \times 80 \times 50$ & $123 \times 28 \times 11 \times 80 \times 50$ & $65 \times 17 \times 8 \times 80 \times 50$ & $96 \times 30 \times 8 \times 80 \times 50$ \\
Second run, minimum cells & $9 \times 6 \times 11 \times 200 \times 50$ & $8 \times 19 \times 11 \times 200 \times 50$ & $5 \times 5 \times 6 \times 200 \times 50$ & $5 \times 18 \times 6 \times 200 \times 50$ \\
Second run, maximum cells & $7 \times 11 \times 8 \times 200 \times 50$ & $9 \times 23 \times 8 \times 200 \times 50$ & $9 \times 9 \times 4 \times 200 \times 50$ & $9 \times 22 \times 4 \times 200 \times 50$ \\
\hline
\end{tabular}

The MASTER code uses $\chi^{2}$ as the figure of merit to choose the best fit, but it is not limited to comparing the models listed in the different catalogues. First, it estimates the reddened SEDs for an interval of $\mathrm{E}(4405-5495)$ between 0 and 3 magnitudes with a number of steps equal to 80 or 200 , depending on the code iteration. In the first phase, we use 80 reddening values between 0 and 3 , including a given extinction law. For each reddened model we estimate 50 distorted SEDs. To do this, we use Monte Carlo, by zeromean Gaussian noise and dispersion, the value of the photometric noise considered in each case. We estimate the best $\chi^{2}$ for each model and, subsequently, we choose the best of all the fitted models (iteration one: path in Fig. 9 marked by blue arrows). With this first solution $\left(T_{\text {eff }}^{1}\right)$, we repeat the process with two fundamental variants: we restrict it to those models with temperatures between $T_{\text {eff }}^{1} \pm 15$ per cent $\cdot T_{\text {eff }}^{1}$ and increase the reddening steps to 200 . We repeat the process until again obtaining the best fit, which we believe to be the best solution (iteration two: path in Fig. 9 marked by green arrows). The generation by means of the Monte Carlo method of 50 samplings for each model allows us to obtain a measure of the internal precision of our estimation following a method similar to the bootstrap. The colours are reddened with the extinction law defined by Maíz Apellániz et al. (2014), which is especially valid for regions of massive star formation. In the Table 2, we show the number of cells of each model mesh used in this work, for both the first and the second runs. We chose four combination of models: $\mathrm{K}$ $+\mathrm{T}, \mathrm{K}+\mathrm{T}+\mathrm{Ko}, \mathrm{C}+\mathrm{T}$, and $\mathrm{C}+\mathrm{T}+\mathrm{Ko}$. In the second run, the number of cells is variable depending on the $T_{\text {eff }}$ derived in the first estimate. In the column corresponding to the second run, we list the maximum and minimum number of cells that we can find in each case. The number of cells is estimated as $N_{T_{\text {eff }}} \times N_{\log g} \times N_{[\mathrm{Fe} / \mathrm{H}]} \mathrm{x}$ $N_{E(4405-5495)}$ x $N_{\text {MonteCarlo. }}$.

In this way, for each physical variable we obtain three representative core values: (i) the value corresponding to the best fitting; and (ii) and (iii), the mean and median of each of the 50 Monte Carlo realizations for each model and each step of E(4405-5495), as well as the standard deviation of each of the physical variables. It is evident that the precision in the determination of the physical variables is mainly determined by the size of the $4 \mathrm{D}$ cells of the models. This is due to since we do not perform any interpolation between the models.

\section{THEORETICAL LIBRARIES TEST}

In this section, we will use the $\mathrm{C}$ and $\mathrm{K}$ stellar model catalogues to test MASTER with different scenarios.

\subsection{Reddening free}

The first test that we carry out is to consider one of the theoretical libraries as observational, using the other as a model. The simplest case would obviously be to compare each model with itself. We have performed this experiment with the $\mathrm{K}$ catalogue for four different photometric errors. We do not include the numerical results of
Table 3. Summary of the results obtained using $\mathrm{C}$ and $\mathrm{K}$ libraries as observational input data with different photometric errors in flux. Column labelled Coelho observational means for $\mathrm{C}$ stellar library as input data and $\mathrm{K}$ stellar library as comparison model. Respectively, the same for the column labelled as Kurucz observational. The analysis has been performed for four different photometric errors, corresponding to 0 per cent, 2 per cent, 5 per cent, and 10 per cent in fluxes.

\begin{tabular}{|c|c|c|c|}
\hline $\begin{array}{l}\text { Photometric } \\
\text { error (per cent) }\end{array}$ & Parameter & $\begin{array}{c}\text { Coelho } \\
\text { observational }\end{array}$ & $\begin{array}{c}\text { Kurucz } \\
\text { observational }\end{array}$ \\
\hline \multirow[t]{6}{*}{0} & $\operatorname{diff} T_{\text {eff }}(\mathrm{K})$ & 1 & 1 \\
\hline & std $T_{\text {eff }}(\mathrm{K})$ & 55 & 55 \\
\hline & diff $\log g($ dex $)$ & 0.00 & 0.00 \\
\hline & std $\log g($ dex $)$ & 0.04 & 0.04 \\
\hline & $\operatorname{diff}[\mathrm{Fe} / \mathrm{H}](\mathrm{dex})$ & -0.02 & 0.02 \\
\hline & std $[\mathrm{Fe} / \mathrm{H}](\mathrm{dex})$ & 0.17 & 0.17 \\
\hline \multirow[t]{6}{*}{2} & $\operatorname{diff} T_{\text {eff }}(\mathrm{K})$ & -7 & -1 \\
\hline & $\operatorname{std} T_{\text {eff }}(\mathrm{K})$ & 111 & 111 \\
\hline & diff $\log g($ dex $)$ & 0.00 & 0.00 \\
\hline & std $\log g($ dex $)$ & 0.05 & 0.05 \\
\hline & $\operatorname{diff}[\mathrm{Fe} / \mathrm{H}](\mathrm{dex})$ & -0.01 & -0.01 \\
\hline & std $[\mathrm{Fe} / \mathrm{H}](\mathrm{dex})$ & 0.27 & 0.27 \\
\hline \multirow[t]{6}{*}{5} & $\operatorname{diff} T_{\text {eff }}(\mathrm{K})$ & -25 & -12 \\
\hline & std $T_{\text {eff }}(\mathrm{K})$ & 178 & 184 \\
\hline & diff $\log g($ dex $)$ & 0.00 & 0.00 \\
\hline & std $\log g(\operatorname{dex})$ & 0.22 & 0.20 \\
\hline & $\operatorname{diff}[\mathrm{Fe} / \mathrm{H}](\mathrm{dex})$ & -0.01 & -0.03 \\
\hline & std $[\mathrm{Fe} / \mathrm{H}](\mathrm{dex})$ & 0.35 & 0.35 \\
\hline \multirow[t]{6}{*}{10} & $\operatorname{diff} T_{\text {eff }}(\mathrm{K})$ & -51 & -37 \\
\hline & std $T_{\text {eff }}(\mathrm{K})$ & 243 & 208 \\
\hline & diff $\log g$ (dex) & -0.02 & -0.02 \\
\hline & std $\log g($ dex $)$ & 0.33 & 0.33 \\
\hline & $\operatorname{diff}[\mathrm{Fe} / \mathrm{H}](\mathrm{dex})$ & -0.02 & -0.04 \\
\hline & std [Fe/H] (dex) & 0.39 & 0.39 \\
\hline
\end{tabular}

this test because they are completely similar to those shown in Table 3. First, we consider no reddening, but that there may be different noise values corresponding to 2 per cent, 5 per cent, and 10 per cent relative errors in the fluxes. The next test follows the same structure, introducing different amounts of reddening in the catalogue used as observational. We start by using the K library as observational and $\mathrm{C}$ as a catalogue of models to compare with. We consider the observations and the models free of reddening, thus obtaining bias and precision in the determination of the physical parameters for different input photometric precisions. We then carry out the same experiment, swapping the role of the libraries. The results are presented in Table 3, where we can see how almost identical results are recovered regardless of the catalogue used as the comparison model.

From Fig. 10, we can see that as we increase the noise, we generate a bias in the determination of the effective temperature. This temperature never exceeds $100 \mathrm{~K}$ (measured as the median of the differences) decreasing the precision in determining the effective 

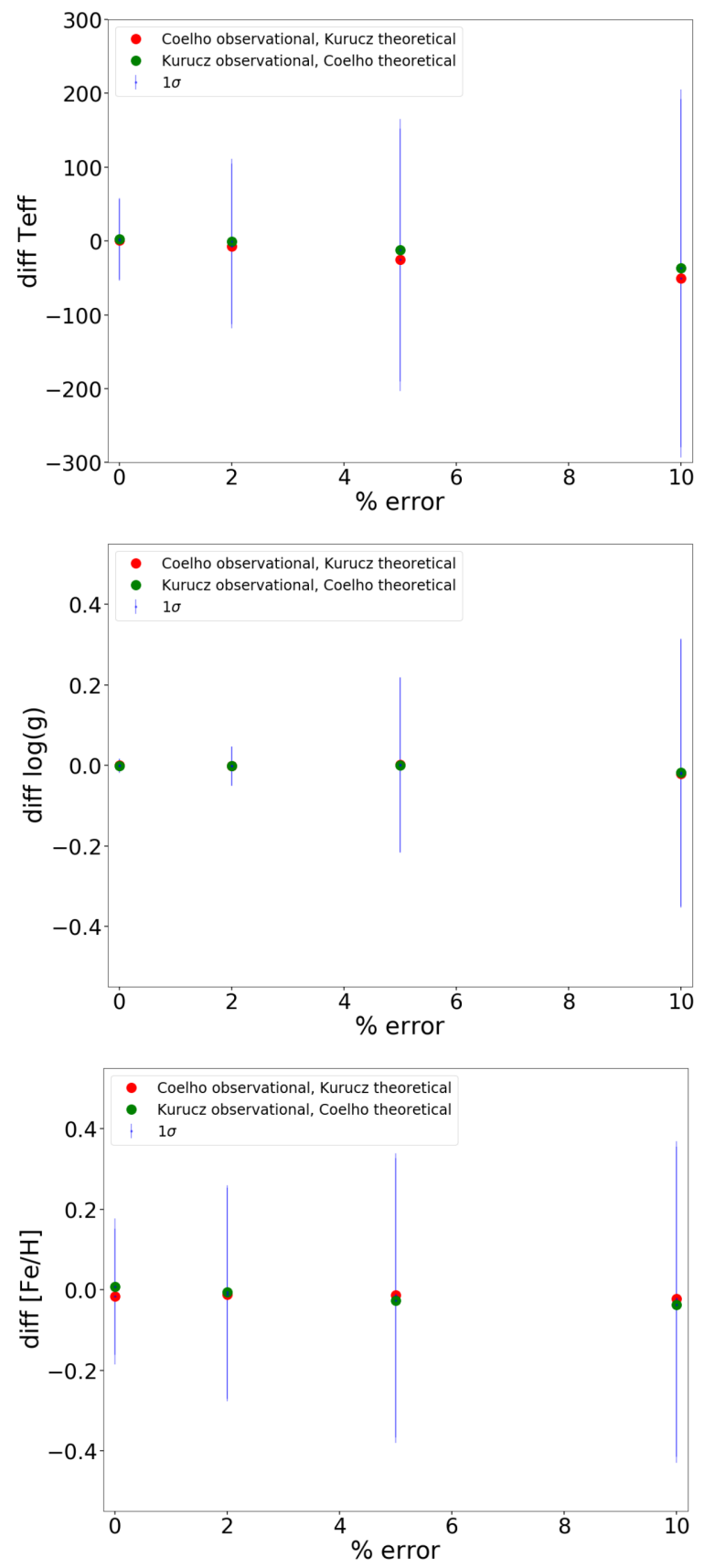

Figure 10. Differences between input and output physical parameters derived by MASTER, for different photometric errors. Red dots are using C library as observational input and $\mathrm{K}$ as comparison models. Green dots refer to the opposite combination ( $\mathrm{K}$ observational and $\mathrm{C}$ as spectral models). Blue lines represent standard deviation as a measurement of the internal precision.

temperature. Although barely perceptible, it seems that the bias is greater when we consider the $\mathrm{K}$ models as the theoretical models and $\mathrm{C}$ as our observational sample. The same happens for $\log g$, but in this case we see how the precision decreases more quickly with the relative error than for the effective temperature. Similar to the metallicities, it seems there is no difference between both libraries used as a comparison model for $\log g$. The top panel shows a systematic deviation to hotter temperatures when we increase photometric errors in the models. None of these errors has an important effect. As we expected, errors increase when we increase observational photometric errors. In the central panel, we observe how the $\log g$ does not show an appreciable bias, instead it shows an internal precision that decreases rapidly with the observational error. Finally, in the bottom panel it is also observed how the metallicity does not seem to be affected by a bias when increasing the photometric errors. Instead, the precision in the determination of this parameter increases with the observational error and is very high $(>0.15)$ in all the cases we analyse.

\subsection{With reddening}

This time we perform the same test but considering different degrees of reddening: in particular, for input we utilize reddening values of $E(4405-5495)$ equal to $0,1,2$, and 3 applied to the library model used as observational data. At the same time, we introduce a grid of extinctions ranging from -1.0 to 4.0 in the models, as explained in Section 2.3. Expanding the reddening range in the set of models avoids edge effect that can appear when the tip values in the models are equal to the tip values of the observational sample. A first performance of this experiment considers a range of $E(4405-5495)$ between 1.0 and 3.0 on the theoretical side. This led to incongruous results that appear to have been overpassed with the new choice of the reddening interval. In this way, we test how our method works by expanding the space of parameters, including extinction. Results of these tests are collected in Table 4.

In this case, the main result is that the introduction of this extrinsic variable increases considerably the bias and the internal error in the determination of the stellar physical parameters. This mainly affects temperatures, where the standard deviation can exceed $1000 \mathrm{~K}$ for reddening of 1-2-3 mag. Table 4 indicates another important points: the collection of reddened stellar models must have reddening that far surpasses the actual reddening of the stars which parameters we want to determine. This is clearly seen in the table when the standard deviations for cases of reddening of 1-2-3 mag increase considerably with respect to 0.0 reddening.

Fig. 11 shows a graphic summary of the results. Again, the red dots represent differences using $\mathrm{C}$ library as observational data and $\mathrm{K}$ as models to compare without Gaussian noise. The blue dots show the differences of the libraries roles swapped. The star markers are those differences applying noise of 5 per cent for models in MASTER. The blue lines are errors in these differences from models without noise, and the red lines for 5 per cent noise.

Fig. 11 shows how the introduction of noise increases the bias in temperature, not reaching more than $200 \mathrm{~K}$ and decreasing the accuracy for both catalogues. This bias in the cases in which we introduce the noise is very similar in each test. The same happens in the estimation of surface gravity, where the bias shows the same behaviour, but with significant errors in the case in which we apply a noise of 5 per cent. In the determination of the metallicity we do not see a bias, regardless of the library or noise we use. Fig. 10 shows how indeterminations in the medians of the differences are close to 0.4 dex. In the case of $E(4405-5495)$, we see how we recover these values without any bias by not introducing noise. However, in the tests with noise there is a slight bias, which is not very pronounced. As far as errors in the determination are concerned, we observe that these are greater in the tests with noise.

\section{OBSERVATIONAL TESTS: NGSL}

We carry out a comparative test of the different stellar models, now using the observational data of the NGSL catalogue. In particular, 
Table 4. The same as in the tests for Table 3. This time we add a new parameter $E(4405-5495)$ in the observational catalogue, applying a grid of reddening in theoretical models of MASTER. The analysis has been performed for four different photometric errors, corresponding to 0 per cent, 2 per cent, 5 per cent, and 10 per cent in fluxes.

\begin{tabular}{|c|c|c|c|c|c|c|c|c|c|}
\hline \multirow[b]{2}{*}{$E(4405-5495)$} & \multirow[b]{2}{*}{ Photometric error (per cent) } & \multicolumn{4}{|c|}{ Coelho observational } & \multicolumn{4}{|c|}{ Kurucz observational } \\
\hline & & 0 & 2 & 5 & 10 & 0 & 2 & 5 & 10 \\
\hline \multirow[t]{8}{*}{0} & $\operatorname{diff} T_{\text {eff }}$ & 10 & 199 & -2140 & 392 & 9 & 208 & -312 & -2180 \\
\hline & $\operatorname{std} T_{\text {eff }}$ & 578 & 1128 & 1686 & 3566 & 573 & 1075 & 1902 & 3560 \\
\hline & diff $\log g$ & -0.02 & 0.04 & -0.06 & -0.24 & -0.02 & 0.04 & -0.07 & -0.26 \\
\hline & std $\log g$ & 0.31 & 0.43 & 0.67 & 0.89 & 0.32 & 0.44 & 0.68 & 0.91 \\
\hline & $\operatorname{diff}[\mathrm{Fe} / \mathrm{H}]$ & -0.01 & 0.02 & -0.01 & -0.10 & 0.02 & 0.01 & -0.01 & -0.10 \\
\hline & std $[\mathrm{Fe} / \mathrm{H}]$ & 0.31 & 0.35 & 0.41 & 0.44 & 0.36 & 0.35 & 0.41 & 0.45 \\
\hline & $\operatorname{diff} E(4405-5495)$ & 0.00 & 0.02 & -0.01 & -0.15 & 0.00 & 0.02 & -0.02 & -0.15 \\
\hline & std $E(4405-5495)$ & 0.06 & 0.07 & 0.08 & 0.22 & 0.07 & 0.07 & 0.11 & 0.22 \\
\hline \multirow[t]{8}{*}{1} & $\operatorname{diff} T_{\text {eff }}$ & 59 & 159 & -325 & -2203 & 62 & 160 & -385 & -2187 \\
\hline & std $T_{\text {eff }}$ & 1024 & 1148 & 1735 & 3635 & 1015 & 1011 & 1679 & 3662 \\
\hline & $\operatorname{diff} \log g$ & 0.05 & 0.06 & -0.07 & -0.24 & -0.03 & 0.07 & -0.08 & -0.27 \\
\hline & std $\log g$ & 0.51 & 0.51 & 0.68 & 0.89 & 0.55 & 0.53 & 0.68 & 0.90 \\
\hline & $\operatorname{diff}[\mathrm{Fe} / \mathrm{H}]$ & 0.00 & 0.03 & 0.00 & -0.10 & -0.02 & 0.02 & -0.02 & -0.10 \\
\hline & $\operatorname{std}[\mathrm{Fe} / \mathrm{H}]$ & 0.42 & 0.39 & 0.42 & 0.44 & 0.42 & 0.39 & 0.41 & 0.44 \\
\hline & $\operatorname{diff} E(4405-5495)$ & 0.01 & 0.02 & -0.02 & -0.15 & 0.00 & 0.01 & -0.02 & -0.16 \\
\hline & std $E(4405-5495)$ & 0.07 & 0.07 & 0.08 & 0.22 & 0.07 & 0.07 & 0.11 & 0.22 \\
\hline \multirow[t]{8}{*}{2} & $\operatorname{diff} T_{\text {eff }}$ & 97 & 191 & -275 & -2156 & 8 & 218 & -335 & -2070 \\
\hline & std $T_{\text {eff }}$ & 908 & 1070 & 1511 & 3520 & 1135 & 1111 & 1798 & 3563 \\
\hline & diff $\log g$ & 0.03 & 0.09 & -0.08 & -0.25 & 0.02 & 0.09 & -0.10 & -0.24 \\
\hline & std $\log g$ & 0.54 & 0.53 & 0.70 & 0.89 & 0.54 & 0.54 & 0.70 & 0.88 \\
\hline & $\operatorname{diff}[\mathrm{Fe} / \mathrm{H}]$ & 0.00 & 0.02 & 0.00 & -0.10 & 0.00 & 0.03 & -0.01 & -0.10 \\
\hline & $\operatorname{std}[\mathrm{Fe} / \mathrm{H}]$ & 0.43 & 0.38 & 0.41 & 0.44 & 0.43 & 0.38 & 0.41 & 0.45 \\
\hline & diff $E(4405-5495)$ & 0.01 & 0.02 & -0.02 & -0.15 & 0.00 & 0.01 & -0.02 & -0.15 \\
\hline & std $E(4405-5495)$ & 0.06 & 0.07 & 0.08 & 0.22 & 0.06 & 0.07 & 0.11 & 0.22 \\
\hline \multirow[t]{8}{*}{3} & $\operatorname{diff} T_{\text {eff }}$ & 6 & 281 & -349 & -2109 & 30 & 236 & -241 & -2185 \\
\hline & std $T_{\text {eff }}$ & 1114 & 1139 & 1872 & 3598 & 1126 & 1096 & 1573 & 3687 \\
\hline & diff $\log g$ & -0.03 & 0.09 & -0.07 & -0.26 & -0.01 & 0.09 & -0.07 & -0.27 \\
\hline & std $\log g$ & 0.56 & 0.44 & 0.67 & 0.89 & 0.56 & 0.40 & 0.67 & 0.91 \\
\hline & $\operatorname{diff}[\mathrm{Fe} / \mathrm{H}]$ & -0.03 & 0.03 & 0.01 & -0.10 & 0.00 & 0.02 & 0.00 & -0.10 \\
\hline & std $[\mathrm{Fe} / \mathrm{H}]$ & 0.48 & 0.36 & 0.41 & 0.44 & 0.47 & 0.35 & 0.40 & 0.44 \\
\hline & $\operatorname{diff} E(4405-5495)$ & 0.00 & 0.02 & -0.02 & -0.15 & 0.00 & 0.01 & -0.01 & -0.16 \\
\hline & std $E(4405-5495)$ & 0.09 & 0.08 & 0.11 & 0.22 & 0.08 & 0.07 & 0.10 & 0.22 \\
\hline
\end{tabular}

we analyse the theoretical stellar libraries of $\mathrm{C}, \mathrm{K}, \mathrm{T}$, and $\mathrm{Ko}$ combining them in the following way: $\mathrm{C}+\mathrm{T}, \mathrm{C}+\mathrm{T}+\mathrm{Ko}, \mathrm{K}+$ $\mathrm{T}$, and $\mathrm{K}+\mathrm{T}+\mathrm{Ko}$. The $\mathrm{T}$ library has been introduced in all cases because it extends the range of effective temperatures catalogued in both $\mathrm{C}$ and $\mathrm{K}$. We have also introduced the $\mathrm{K}$ library to take into account the WDs that could be found in certain regions of the Galactic disc. Additionally, it can be observed how its use combined with the other stellar libraries could even introduce artefacts in the determination of stellar physical parameters. This can be checked using the NGSL library that does not contain any catalogued WD.

The maximum colour excess catalogued in the NGSL library does not exceed $0.80 \mathrm{mag}$. Here, we introduce reddening in the theoretical stellar models that reaches up to $3 \mathrm{mag}$ to avoid as far as possible the effect of the edges discussed in Section 3. We will assume that the NGSL has a typical error of 0.05 mag for all colours.

\subsection{NGSL test with $K+T$ theoretical libraries}

We begin by using the $\mathrm{K}$ and $\mathrm{T}$ theoretical stellar libraries. Fig. 12 represents the models of $\mathrm{K}+\mathrm{T}$ in the plane of the physical variables $T_{\text {eff }}$ and $\log g$ including also the observational NGSL catalogue.
There we observe that there are five NGSL stars which labelled temperatures are not covered by the model grid.

These are the stars: LHS10, HD 123657, HD 126327, HD 175865 , and VGKCOM, all of them with $T_{\text {eff }}<3500 \mathrm{~K}$. Three of these five stars are labelled as binary or suspect, and the other two have an extremely high $\chi^{2}$ but with a $T_{\text {eff }}=3500 \mathrm{~K}$ which is the lowest temperature value in the models. For HD 123657 , with a much smaller $\chi^{2}$ (7.4) we obtain a similar $T_{\text {eff }}$ to the one catalogued. The differences lie in the estimation of the $\log g$; in the case of LHS10, it is 5.34 dex in the library, while we obtain 2.5 dex. In the case of HD 123657, it is tabulated with a value of 0.59 dex and we obtain it with 0.5 dex.

In Fig. 13(a), we represent the results obtained for the effective temperatures, colouring them according to their $\log g$. The green line serves as a 1:1 visual reference between both values. In this case, we see a high degree of conformity between the values catalogued and those estimated from these models for the effective temperature, slightly increasing the dispersion for the OB stars. The dots density map seems to fit very well to the 1:1 line and the median of the difference of the fitted stars is of the order of 3 per cent in effective temperature with its standard deviation not exceeding 11 per cent. Another remarkable fact is the deviation towards hot temperatures of three stars not considered as binary or suspect 

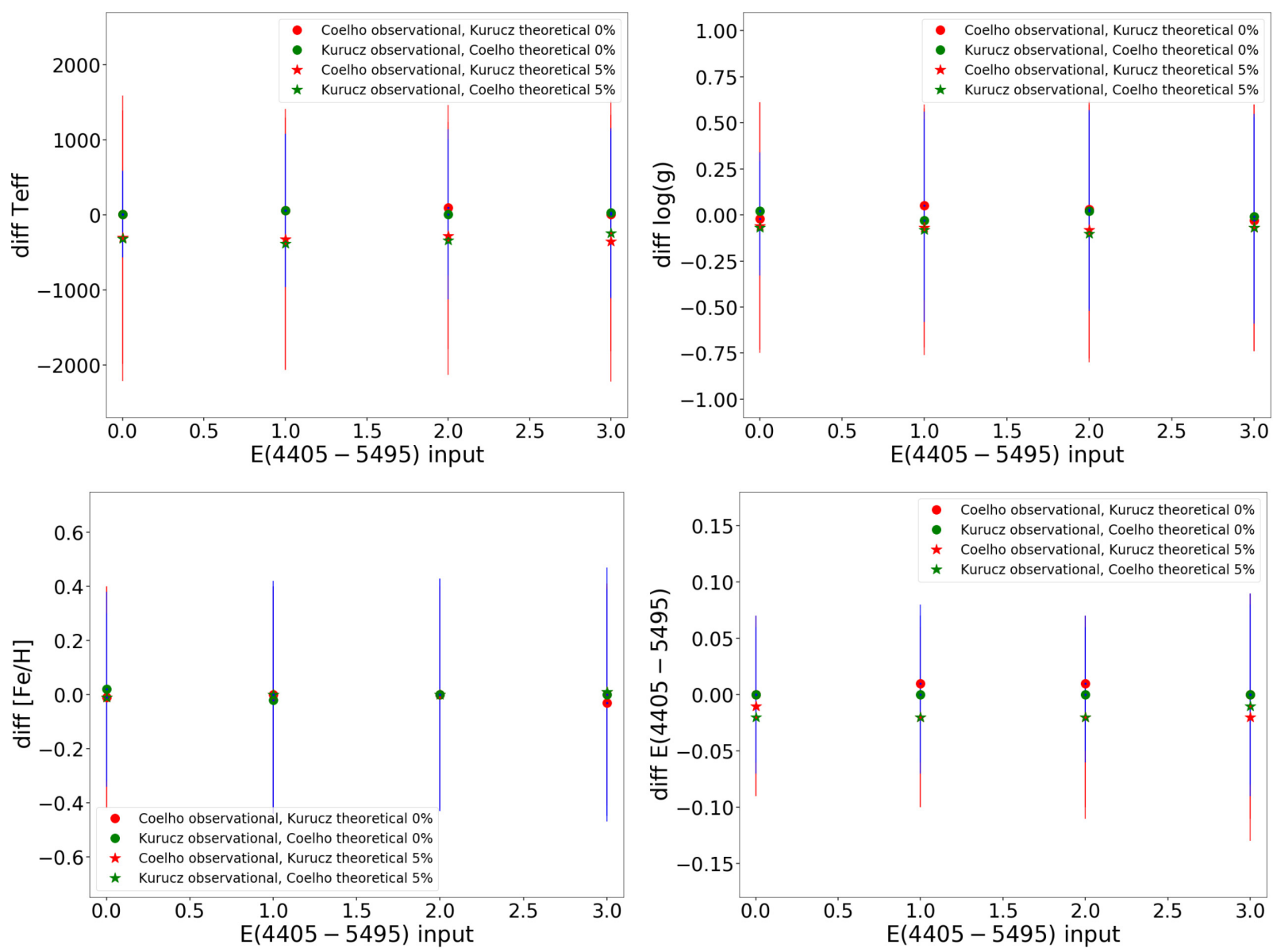

Figure 11. Graphic summary of the results listed in Table 4. Colour dots represent models without noise, while colour stars show results using a 5 per cent photometric error in flux, introducing different values of reddening in the input catalogue. Blue lines refer to standard deviation in the absence of photometric error, while red lines correspond to a 5 per cent photometric error in flux.

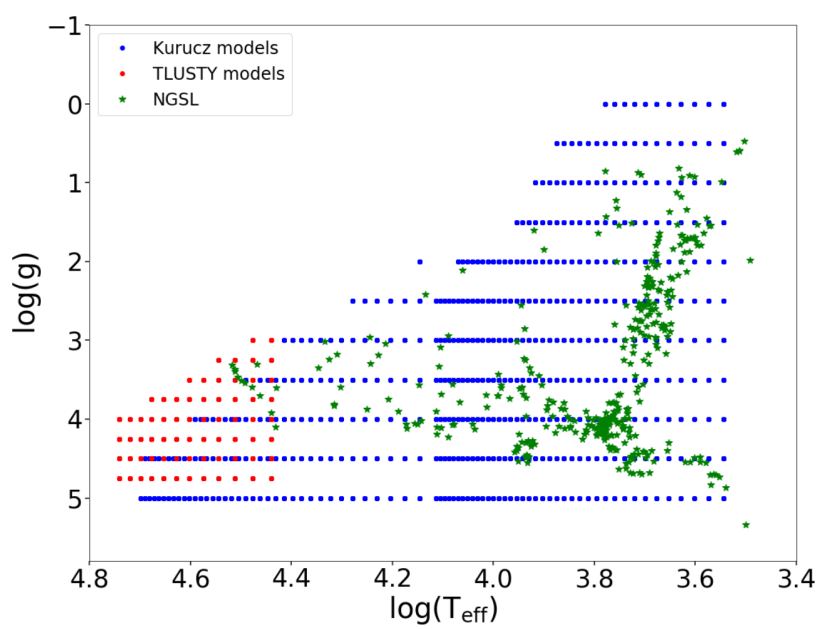

Figure 12. Dots represent $\mathrm{K}+\mathrm{T}$ theoretical stellar models in the plane of the physical variables $T_{\text {eff }}$ and $\log g$. The observational NGSL catalogue is drawn in green stars.

[in ascending order of $T_{\text {eff }}$ they are BD 174708 (binary according to SIMBAD), HD 142703 (variable star according to SIMBAD, gamma-Bootis star), and HD 111786 (variable star according to SIMBAD of delta Sct type)]. Star HD 126327 has a catalogued temperature of $3100 \mathrm{~K}$ and with a surface gravity of $1.98 \mathrm{dex}$, which is not covered by this grid of models. This leads to an adjustment with a $\chi^{2}$ above the hundred and very erroneous values in all physical parameters except $T_{\text {eff }}$, for which we obtain a value of $3500 \mathrm{~K}$.

The results in Fig. 13(b) are not so good for the determination of the surface gravity where there is a bias defined by the median of the differences. This difference reaches the value of -0.32 dex and mainly affects the giant and supergiant stars, while the MS stars do not present such a remarkable bias and also show a lower dispersion for the whole metallicity range.

The determination of metallicities in Fig. 13(c) shows worse results and is very dependent on temperature. The density map shows that the highest concentration of objects fits the 1:1 line. However, we see how stars with high temperatures (red dots) show chaotic results, tending mainly to determine metallicities much lower than those catalogued. There is a clear bias towards more metallic determinations for metallicities catalogued $<-1.0 \mathrm{dex}$, even for stars of late spectral type. However, the median of the differences is of the order of $-0.04 \mathrm{dex}$, which shields the biases mentioned above.

Finally, in Fig. 13(d), we represent the obtained reddening values. It can be observed how there is a clear tendency (observable in the density map) to overestimate the colour excess determined, although the median of the differences shows a value close to 0.0 . 

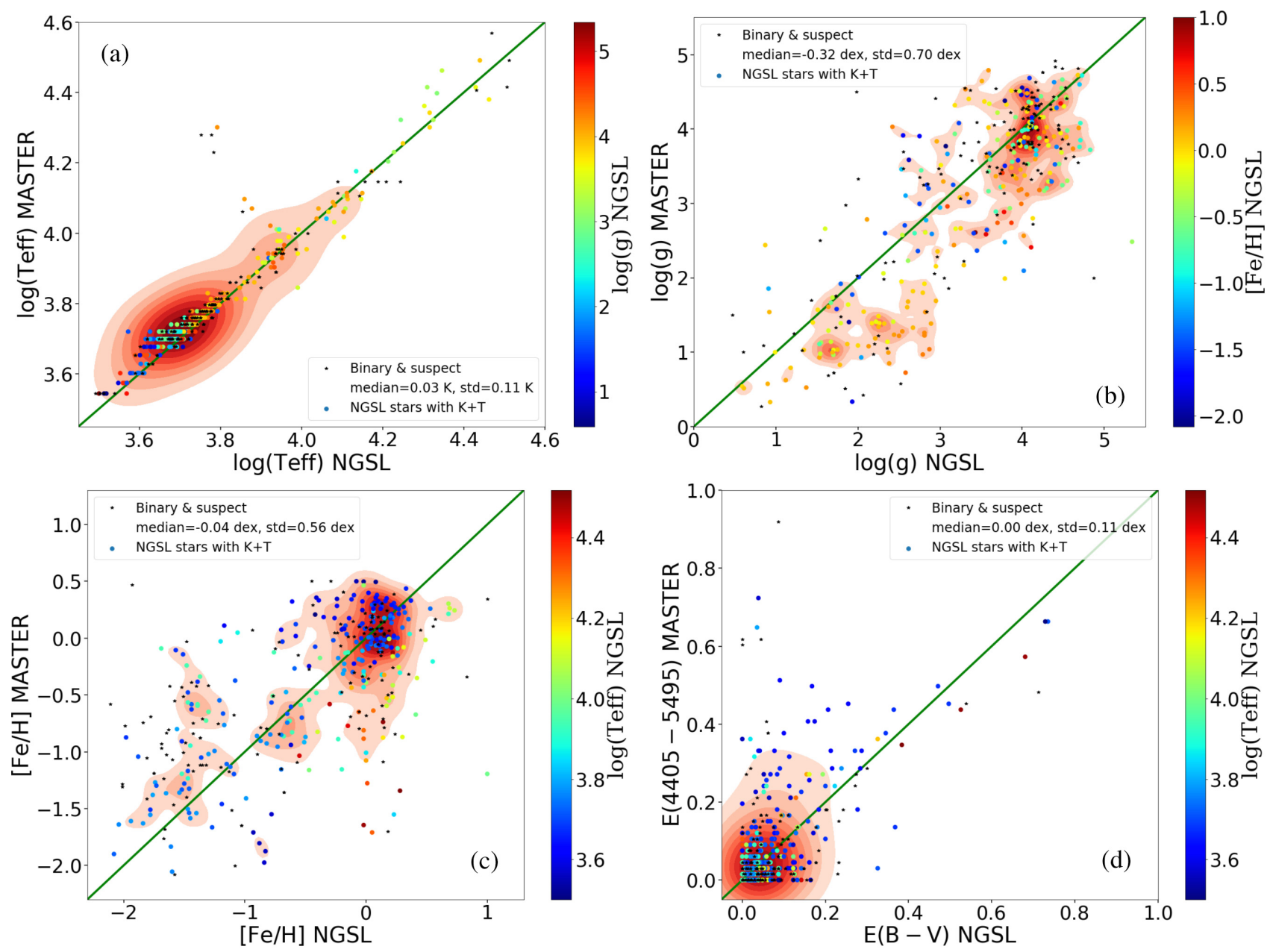

Figure 13. Comparison of (a) $T_{\text {eff }}$, (b) $\log g$, (c) $[\mathrm{Fe} / \mathrm{H}]$, and (d) colour excess for NGSL stars derived from theoretical libraries $\mathrm{K}+\mathrm{T}$ with Koleva \& Vazdekis (2012) values.

\subsection{NGSL test with $\mathrm{C}+\mathrm{T}$ theoretical libraries}

Fig. 14 shows the range of coverage of these $\mathrm{C}+\mathrm{T}$ theoretical stellar models with the observational NGSL library. Blue and red dots represent theoretical catalogues, while green stars are the NGSL catalogue stars. Here, we observe how at least one cold star is outside the coverage, while for OB stars, the grid of models has more remarkable discontinuities than with $\mathrm{K}+\mathrm{T}$. In Fig. 15, we plot this grid of models in a $\log g-[\mathrm{Fe} / \mathrm{H}]$ context. As can be seen, we cannot reproduce the NGSL library in metallicities.

Using $\mathrm{C}+\mathrm{T}$ we find very similar results to those of $\mathrm{K}+\mathrm{T}$. Yet, the differences are smaller for the $\log \left(T_{\text {eff }}\right)$ and the gravity and colour excess, as we can see in Figs 16(a) and (d). However, there is a clear bias in the determination of the metallicities with tabulated values for $[\mathrm{Fe} / \mathrm{H}]<-1.0$ dex. This is due to this grid of models does not cover such low metallicities for temperatures below $25000 \mathrm{~K}$, as we expect from Fig. 15. This fact leads us to obtain erroneous metallicities, so these fittings end up being worse than those obtained with $\mathrm{K}+\mathrm{T}$.

Next, we show the results that we obtain for $\log g$ in Fig. 16(b). Here, we extract again results very similar to those of Fig. 13(b) for MS stars. However, for giant and supergiant stars with solar metallicity or higher, lower $\log g$ values are obtained systematically.

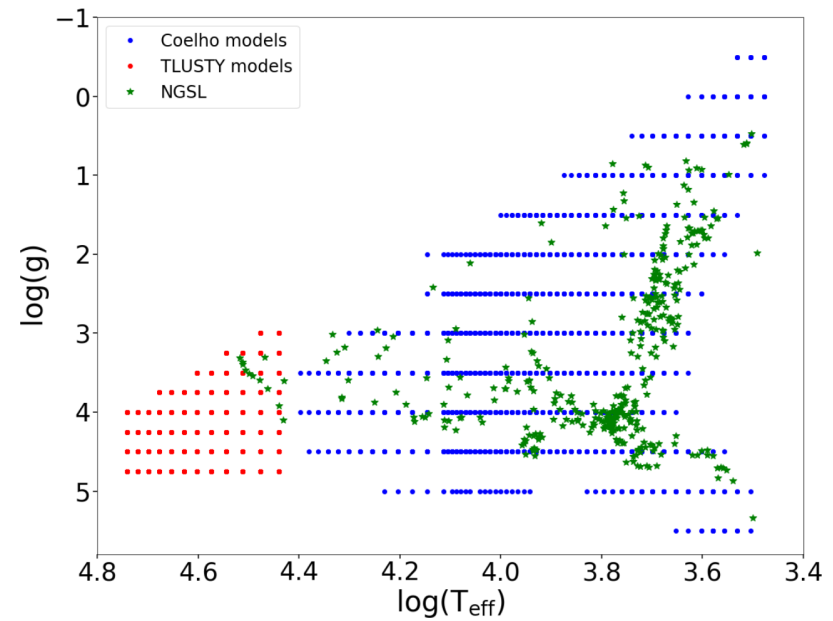

Figure 14. Dots represent $\mathrm{C}+\mathrm{T}$ theoretical stellar models in the plane of the physical variables $T_{\text {eff }}$ and $\log g$. The observational NGSL catalogue is drawn in green stars. 


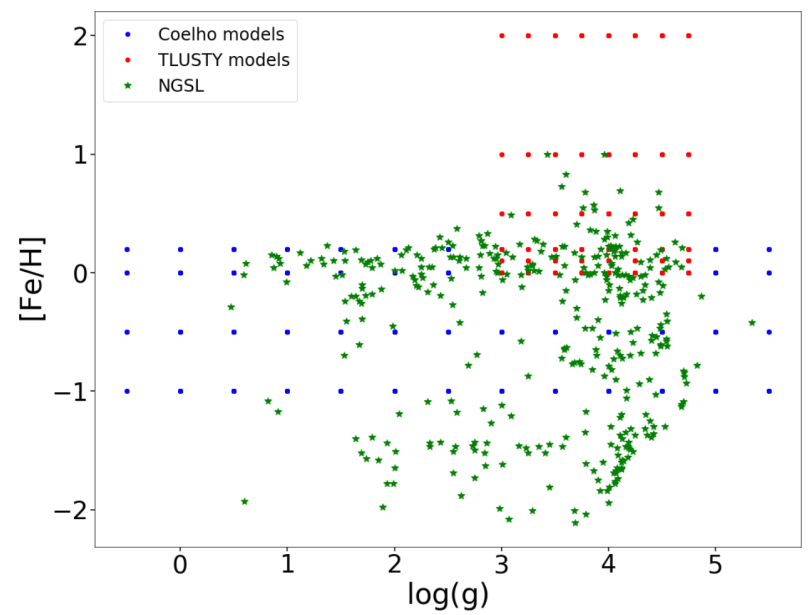

Figure 15. Dots represent $\mathrm{C}+\mathrm{T}$ theoretical stellar models in the plane of the physical variables $\log g$ and $[\mathrm{Fe} / \mathrm{H}]$. The observational NGSL catalogue is drawn in green stars.

In comparison with what was obtained using $\mathrm{K}+\mathrm{T}$, differences are obtained in similar values but with a higher deviation.

We continue with Fig. 16(c), which represents the results of $[\mathrm{Fe} / \mathrm{H}]$. In this case, the results that we obtain are concentrated between -1.0 and 0.0 dex for non-OB stars. This happens due to the coverage of the $\mathrm{C}$ models have a best fitting for this type of stars, while for very hot stars, we have the best fitting with the $\mathrm{T}$ models. This fact indicates that the coverage of the $\mathrm{C}$ models with $[\alpha / \mathrm{Fe}]=0.0$ for the metallicity is not complete. Thus, the results are systematically diverted towards incorrect $[\mathrm{Fe} / \mathrm{H}]$ values.

Finally, we see the results of colour excess for this study with this pair of models represented in Fig. 16(d). Here, we observe again the fact of the coupling between temperature and colour excess. A bad determination of one of them entails not correctly deriving the other. In this case, in Fig. 16 the number of NGSL stars with a poorly obtainded temperature is larger than in Fig. 13 using other models. For this reason, there are more objects with a wrong E(4405-5495). We can therefore conclude that, for the same objects of the NGSL library, the theoretical stellar models that work best in deriving their physical properties with MASTER are $\mathrm{K}+\mathrm{T}$.

\subsection{NGSL test adding Ko theoretical libraries}

Fig. 17 shows the GALANTE normalized colours of different models with $T_{\text {eff }}=30000 \mathrm{~K}$ for different surface gravity values. In the case of the MS models, the GALANTE colours are almost indistinguishable from the WD model for $\log g=6.5$. This indistinguishability between MS and WD models increases as the
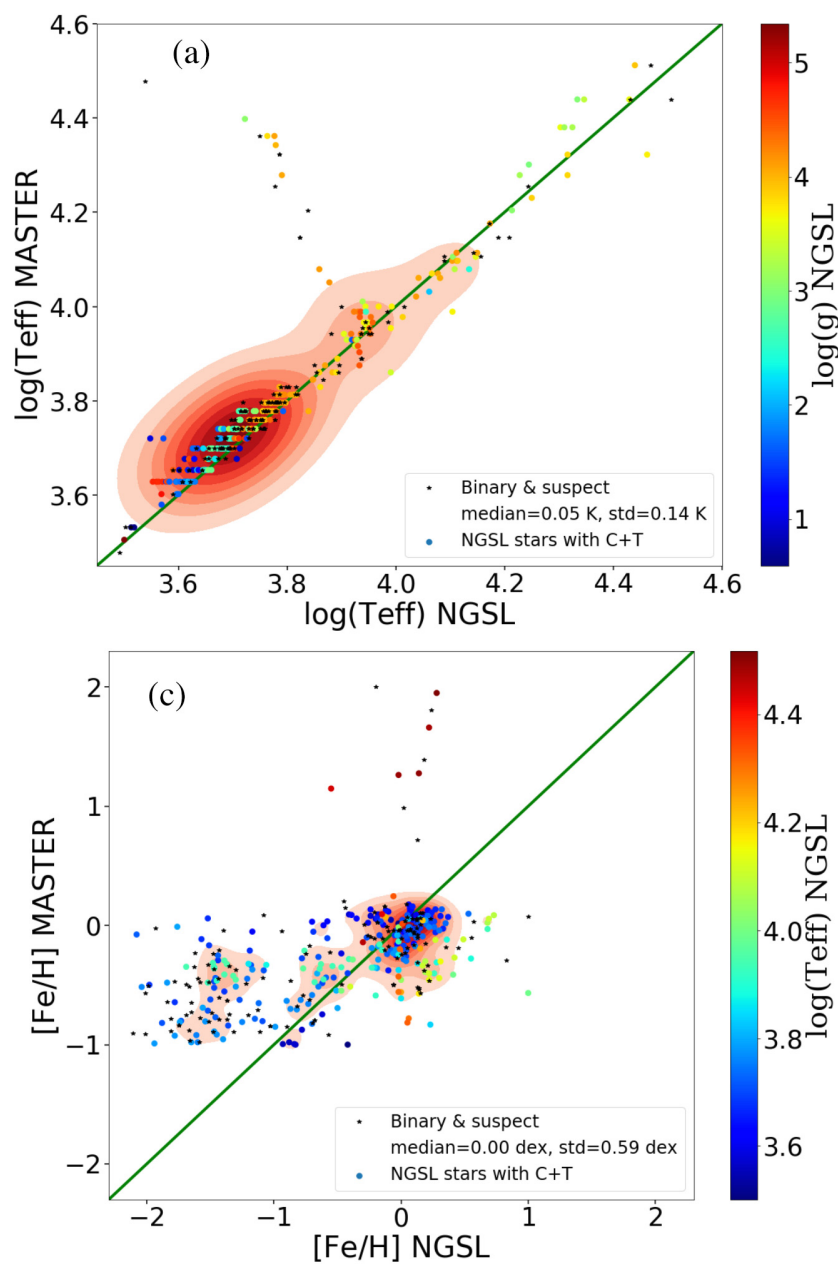
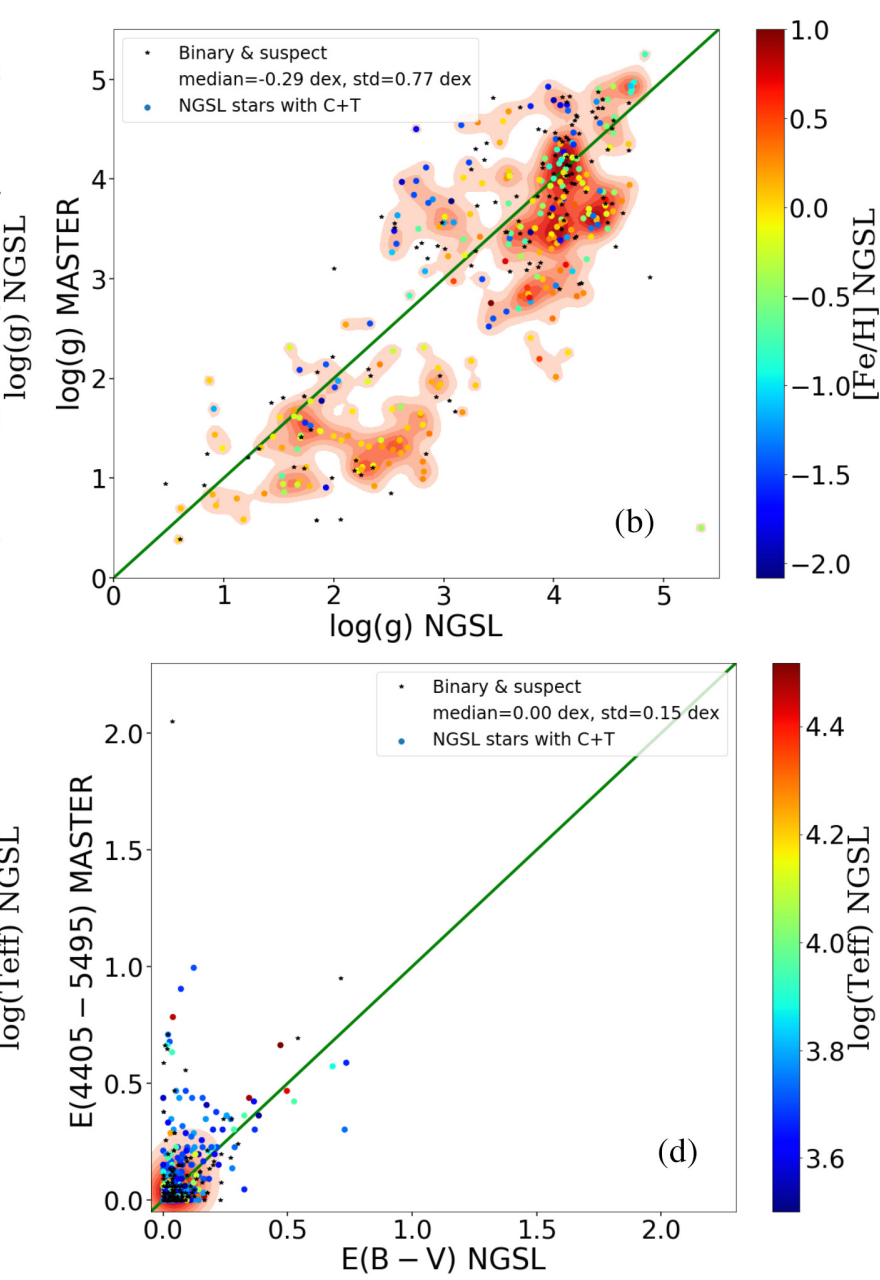

Figure 16. Comparison of (a) $T_{\text {eff }}$, (b) $\log g$, (c) [Fe/H], and (d) colour excess for NGSL stars derived from theoretical libraries C + T with Koleva \& Vazdekis (2012) values. 


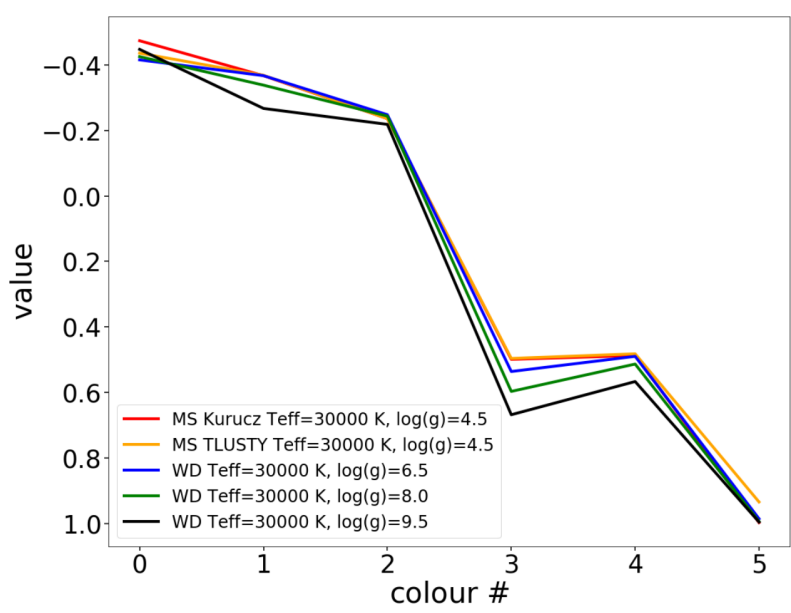

Figure 17. GALANTE normalized colours of different models with $T_{\text {eff }}=30000 \mathrm{~K}$ for different surface gravity values.

temperature increases. Thus, we must be careful in the choice of models for the estimation of physical parameters, since they could at some point determine more WDs than those that really exist in the population we are observing.
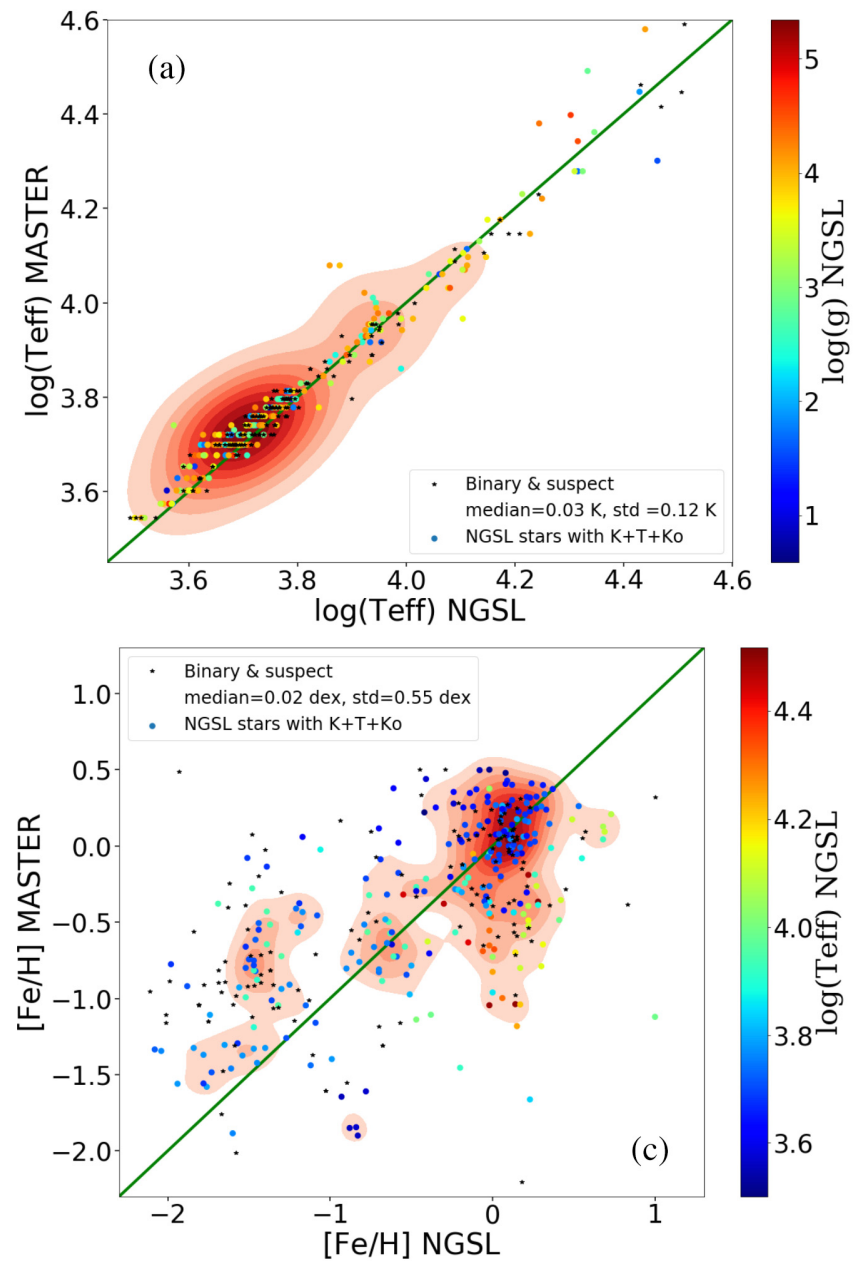

Once we have carried out the study of NGSL with these pairs of theoretical libraries, we wonder whether introducing a third theoretical catalogue would introduce some kind of degeneration when selecting the best model after applying reddening in the GALANTE normalized colours. In order to carry out this study, we select the catalogue of Ko WD models.

Following the same order, in Fig. 18(a) we represent the $T_{\text {eff }}$ derived with MASTER using the $\mathrm{K}+\mathrm{T}+\mathrm{Ko}$ models. If we compare these results with those from Fig. 13, we see that the temperatures are very similar except for those stars that deviated from the correct ones between 3.8 and $4.0 \log \left(T_{\text {eff }}\right)$. In this occasion, the models to which they fit best are somewhat colder. On the other hand, OB stars are derived with values similar to those in Fig. 13. This yields a pair of stars with some $\log g$ from supergiants and not from giants. We can conclude from this comparison that those cold stars with difficulties have improved slightly when determining their $T_{\text {eff }}$. It should be noted that this has not happened when introducing WD models: no object shows a $\log g$ of these kind of stars, showing the robustness of the method with the appropriate catalogues.

Fig. 18(b) shows the results in $\log g$. When comparing with Fig. 13(b) we observe a very similar distribution to that obtained with the $\mathrm{K}+\mathrm{T}$ models. As expected, here we can see that the derived values of $\log g$ come only from the models of $\mathrm{K}+\mathrm{T}$, without using those from WD models.

Figure 18. Comparison of (a) $T_{\text {eff }}$, (b) $\log g$, (c) $[\mathrm{Fe} / \mathrm{H}]$, and (d) colour excess for NGSL stars derived from theoretical libraries $\mathrm{K}+\mathrm{T}+\mathrm{Ko}$ with Koleva $\&$ Vazdekis (2012) values. 

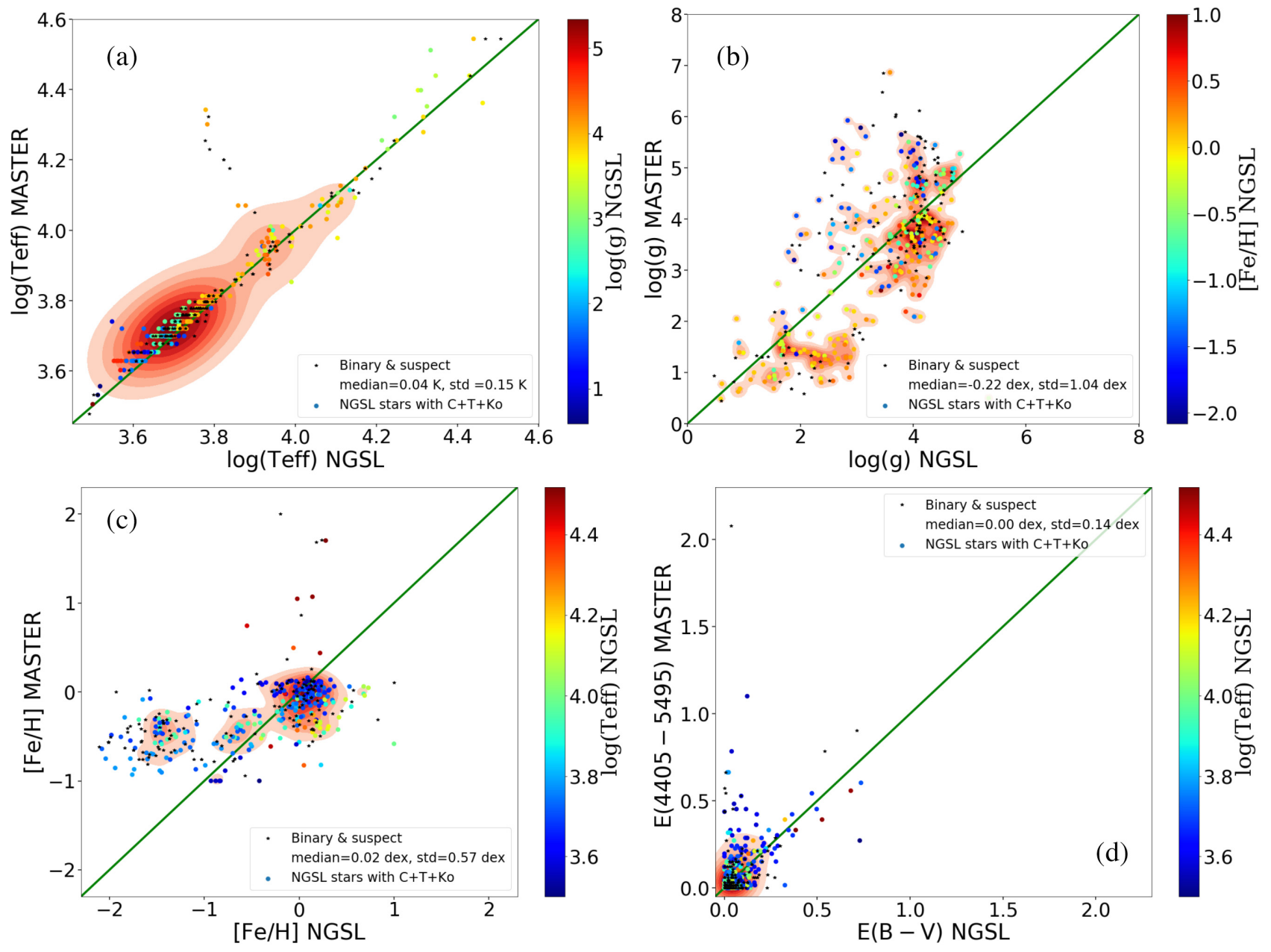

Figure 19. Comparison of (a) $T_{\text {eff }}$, (b) $\log g$, (c) $[\mathrm{Fe} / \mathrm{H}]$, and (d) colour excess for NGSL stars derived from theoretical libraries $\mathrm{C}+\mathrm{T}+\mathrm{Ko}$ with Koleva \& Vazdekis (2012) values.

Table 5. This table summarizes the results we obtain for each block of models using the NGSL as input catalogue. The term $\operatorname{diff} E(B-V)$ means for the difference between the $E(B-V)$ listed in Koleva \& Vazdekis (2012) and the $E(4405-5495)$ estimated by MASTER. This difference has an intrinsic component depending on actual temperature and reddening (Maíz Apellániz 2013a).

\begin{tabular}{lcccc}
\hline & $\mathrm{K}+\mathrm{T}$ & $\mathrm{K}+\mathrm{T}+\mathrm{Ko}$ & $\mathrm{C}+\mathrm{T}$ & $\mathrm{C}+\mathrm{T}+\mathrm{Ko}$ \\
\hline diff $\log \left(T_{\text {eff }}\right)$ & 0.03 & 0.03 & 0.05 & 0.04 \\
std diff $\log \left(T_{\text {eff }}\right)$ & 0.11 & 0.12 & 0.14 & 0.15 \\
diff $\log g$ & -0.32 & -0.22 & -0.29 & -0.22 \\
std diff $\log g$ & 0.70 & 0.89 & 0.77 & 1.04 \\
diff $[\mathrm{Fe} / \mathrm{H}]$ & -0.04 & 0.02 & 0.00 & 0.02 \\
std diff $[\mathrm{Fe} / \mathrm{H}]$ & 0.56 & 0.55 & 0.59 & 0.57 \\
diff $E(B-V)$ & 0.00 & 0.00 & 0.00 & 0.00 \\
std diff $E(B-V)$ & 0.11 & 0.11 & 0.15 & 0.14 \\
$\chi^{2}$ (per cent $)<5$ & 93 & 93 & 92 & 92 \\
$\chi^{2}$ (percent $)<2$ & 80 & 80 & 77 & 77 \\
\hline
\end{tabular}

In terms of metallicities, Fig. 18(c) presents the results we obtain using the three catalogues in MASTER. The results in the distribution of densities are very similar. Yet, the values that are not correctly recovered are distributed further away from those tabulated by the authors, which explains the deviation we derive in this case.
Finally, we do not expect any change in the values of $E$ (4405-5495). This is due to the $T_{\text {eff }}$ have not been derived from the Ko models. Fig. 18(d) represents the colour excess values for this set of three models. In this parameter, the values that we obtain are exactly those expected when launching MASTER using only the $\mathrm{K}+\mathrm{T}$ models, confirming the certainty of our results.

We will now present the MASTER results for the NGSL stars, inputting models of $\mathrm{C}+\mathrm{T}$ into MASTER with the addition of the Ko WD models. In this case, the inclusion of the Ko models can cause some of these results to improve their fitting from the WD models. This is due to the fact that $T_{\text {eff }}$ with the $\mathrm{C}+\mathrm{T}$ were not derived as correctly as using the $\mathrm{K}+\mathrm{T}$ libraries. Fig. 19 presents all the results for each physical parameter using these models in MASTER.

In this case, we see an improvement that is not entirely correct in the $T_{\text {eff }}$ for cold stars. This improvement, as we can see in the results of the $\log g$ in the plot (b), comes from a better fit using WD models. It should be remembered that, among the NGSL stars, there is not any catalogued star of this type. Thus, it is attaining a better mathematical solution of $\chi^{2}$, but without being a better physical solution.

Regarding metallicity, this physical variable is still not recovered correctly. This is due to the $\mathrm{C}$ models do not cover this parameter for the range of values that the NGSL library has. Again, erroneous values are derived for those stars that best adjust using $\mathrm{K}$ when forced to obtain a better $\chi^{2}$ mathematical result. 
Finally, we observe a somewhat higher value in those stars that have better fitting to some WD models. However, in Fig. 19(d), it can be observed that this only happens for those stars for which this theoretical catalogue was used to get a better solution in their fitting. This fact reflects the correlation between the estimation of the effective temperature of a star and its reddening. Therefore, if the best fitting is obtained from a WD model, its colours are reddened with a higher E(4405-5495) value to get its modified GALANTE colours for the model until it matches the GALANTE colours for NGSL.

Table 5 summarizes the results we obtain for each block of models using the NGSL as an observational catalogue. We also introduce the percentages of the NGSL stars that we obtain with $\chi^{2}$ below 5 per cent and 2 per cent. In view of these results, we can conclude that the set of catalogues of $\mathrm{K}+\mathrm{T}$ obtains more accurate results in reference to those catalogued for these stars of the NGSL library.

We include the results of the derivation of the physical stellar parameters of the NGSL stars from the $\mathrm{K}+\mathrm{T}$ models as a complementary table (Table A1).

\section{SUMMARY AND CONCLUSIONS}

In this paper, we analyse the differences between several theoretical stellar libraries. The result is that, for $T_{\text {eff }}<10000 \mathrm{~K}$, there are discrepancies between them, highlighting a dependence on the catalogue used. We also analysed with MASTER a fitting of models to observational SEDs developed by us and based on $\chi^{2}$. The behaviour of the different models is studied as well as how to work better with them to obtain a lower bias and the inclusion of artefacts. To do this, we use the GALANTE normalized colours and perform different tests between the models, both for the case of extinction and without it. Here, we see how the inclusion of this extrinsic parameter has great weight, showing an increase both in the medians of the differences and standard deviations in comparison to the same study carried out without applying extinctions. Once this analysis with models was done, we used the NGSL observational catalogue, using three libraries of models with extinction. After analysing the results, we expanded our grid of models with a WD catalogue. Thus, we checked whether our method is sufficiently robust when introducing spectral models that are not catalogued in the NGSL. For the specific case of $\mathrm{C}+\mathrm{T}$, we see that the introduction of this library causes a couple of stars to adjust better with WD SEDs. The bad fitting $\chi^{2}$ obtained from this group of models improves with the inclusion of the Ko models, due to the impossibility of covering all the physical parameters of the NGSL with the $\mathrm{C}+\mathrm{T}$ models.

\section{ACKNOWLEDGEMENTS}

We thank the Centro de Estudios de Física del Cosmos de Aragón (CEFCA) team in Teruel and Javalambre for supporting us in this project, giving us the opportunity to use non-J-PLUS useful nights to develop the GALANTE survey. We also want to recognize the work of the NGSL team (Gregg et al. 2006; Heap \& Lindler 2016) whose observational catalogue has been of great help for our work. This research made use of PYTHON (http://www.python.org); NUMPY (van der Walt, Colbert \& Varoquaux 2011); SCIPY (Jones et al. 2001); and MATPLOTLIB (Hunter 2007), a suite of open-source PYTHON modules that provides a framework for creating scientific plots. We also acknowledge the use of STILTS and TOPCAT tools (Taylor 2005). AL-G, EJA, and JMA acknowledge support from the Spanish Government Ministerio de Ciencia, Innovación y Universidades though grants AYA2013-40 611-P and AYA2016-75 931-C2-1/2-P. AL-G and EJA also acknowledge support from the State Agency for
Research of the Spanish MCIU through the 'Center of Excellence Severo Ochoa' award for the Instituto de Astrofísica de Andalucía (SEV-2017-0709).

\section{REFERENCES}

Allende Prieto C., 2016, A\&A, 595, A129

Allende Prieto C., del Burgo C., 2016, MNRAS, 455, 3864

Aparicio Villegas T. et al., 2010, AJ, 139, 1242

Barlow R., 1989, Statistics. A Guide to the Use of Statistical Methods in the Physical Sciences, Wiley, New York

Bohlin R. C., Mészáros S., Fleming S. W., Gordon K. D., Koekemoer A. M., Kovács J., 2017, AJ, 153, 234

Castelli F., Gratton R. G., Kurucz R. L., 1997, A\&A, 318, 841

Coelho P. R. T., 2014, MNRAS, 440, 1027

Gregg M. D. et al., 2006, in Koekemoer A. M., Goudfrooij P., Dressel L. L., eds, The 2005 HST Calibration Workshop: Hubble After the Transition to Two-Gyro Mode, p. 209

Heap S. R., Lindler D., 2016, in Deustua S., Allam S., Tucker D., Smith J. A., eds, ASP Conf. Ser. Vol. 503, The Science of Calibration. Astron. Soc. Pac., San Francisco, p. 211

Hunter J. D., 2007, Comput. Sci. Eng., 9, 90

Jones E. et al., 2001, SciPy: Open Source Scientific Tools for Python. Available at: http://www.scipy.org/

Koester D., 2010, Mem. Soc. Astron. Ital., 81, 921

Koleva M., Vazdekis A., 2012, A\&A, 538, A143

Kurucz R. L., 1970, SAO Special Report, p. 309

Kurucz R. L., 2005, Mem. Soc. Astron. Ital. Suppl., 8, 14

Lanz T., Hubeny I., 2003, ApJS, 146, 417

Lorenzo-Gutiérrez A. et al., 2019, MNRAS, 486, 966

Maíz Apellániz J., 2013a, in Guirado J. C., Lara L. M., Quilis V., Gorgas J., eds, Highlights of Spanish Astrophysics VII. p. 583

Maíz Apellániz J., 2013b, in Guirado J. C., Lara L. M., Quilis V., Gorgas J., eds, Highlights of Spanish Astrophysics VII. p. 657

Maíz Apellániz J., 2017, in Early Data Release and Scientific Exploitation of the J-PLUS Survey. Teruel, Spain, p. 15

Maíz Apellániz J., Barbá R. H., 2018, A\&A, 613, A9

Maíz Apellániz J., Sota A., 2008, Rev. Mex. Astron. Conf., 33, 44

Maíz Apellániz J. et al., 2014, A\&A, 564, A63

Maíz Apellániz J. et al., 2019, in Montesinos B., Asensio Ramos A., Buitrago F., Schödel R., Villaver E., Pérez-Hoyos S., Ordóñez-Etxeberria I., eds, Highlights on Spanish Astrophysics X. p. 346

Mann A. W., von Braun K., 2015, PASP, 127, 102

Rodrigo C., Solano E., Bayo A., Cortés-Contreras M., Jiménez-Esteban F., 2019, in Montesinos B., Asensio Ramos A., Buitrago F., Schödel R., Villaver E., Pérez-Hoyos S., Ordóñez-Etxeberria I., eds, Highlights on Spanish Astrophysics X. p. 430

Taylor M. B., 2005, in Shopbell P., Britton M., Ebert R., eds, ASP Conf. Ser. Vol. 347, Astronomical Data Analysis Software and Systems XIV. Astron. Soc. Pac., San Francisco,p. 29

Weiler M., 2018, A\&A, 617, A138

van der Walt S., Colbert S. C., Varoquaux G., 2011, Comput. Sci. Eng., 13, 22

\section{SUPPORTING INFORMATION}

Supplementary data are available at MNRAS online.

Table A1. Comparison of example stars covering the range of each stellar physical parameters of the NGSL empirical library.

Please note: Oxford University Press is not responsible for the content or functionality of any supporting materials supplied by the authors. Any queries (other than missing material) should be directed to the corresponding author for the article.

APPENDIX A: NGSL TABLE 
Table A1. Comparison of example stars covering the range of each stellar physical parameters of the NGSL empirical library. We tabulate nine NGSL stars by way of example. We will include the full NGSL catalogue in the electronic version.

\begin{tabular}{lrrrrrrrr}
\hline $\begin{array}{l}\text { NGSL } \\
\text { star }\end{array}$ & $\begin{array}{c}\text { Input } \\
T_{\text {eff }}(\mathrm{K})\end{array}$ & $\begin{array}{c}\text { Output } \\
T_{\text {eff }}(\mathrm{K})\end{array}$ & $\begin{array}{c}\text { Input } \\
\log g\end{array}$ & $\begin{array}{c}\text { Output } \\
\log g\end{array}$ & $\begin{array}{c}\text { Input } \\
{[\mathrm{Fe} / \mathrm{H}]}\end{array}$ & $\begin{array}{c}\text { Output } \\
{[\mathrm{Fe} / \mathrm{H}]}\end{array}$ & $\begin{array}{c}\text { Input } \\
E(B-V)\end{array}$ & $\begin{array}{c}\text { Output } \\
E(4405-5495)\end{array}$ \\
\hline HD 126 327 & 3100 & 3500 & 1.98 & 4.50 & -0.45 & 0.50 & 0.09 & 0.87 \\
BD 442 051 & 3664 & 3750 & 4.70 & 4.50 & -0.83 & -1.82 & 0.06 & 0.05 \\
BD 511 696 & 5746 & 6000 & 4.39 & 4.50 & -1.25 & -1.21 & 0.03 & 0.04 \\
HD 111 786 & 7549 & 10000 & 4.17 & 3.50 & -1.06 & -0.39 & 0.03 & 0.15 \\
HD 143 459 & 10298 & 9250 & 3.85 & 3.00 & -0.47 & -0.90 & 0.13 & 0.03 \\
HD 212 593 & 13642 & 14000 & 2.42 & 3.00 & 0.30 & -0.27 & 0.16 & 0.20 \\
HD 175 156 & 16361 & 17000 & 3.04 & 3.00 & 0.17 & -0.37 & 0.33 & 0.37 \\
HD 096 446 & 20086 & 24000 & 3.59 & 4.00 & 0.06 & -0.74 & 0.07 & 0.07 \\
HD 057 061 & 32514 & 27000 & 3.37 & 4.00 & 0.18 & -2.42 & 0.20 & 0.10 \\
\hline
\end{tabular}

This paper has been typeset from a $\mathrm{T}_{\mathrm{E}} \mathrm{X} / \mathrm{L} \mathrm{T}_{\mathrm{E}} \mathrm{X}$ file prepared by the author. 